\title{
Quantum-Zeno Fermi polaron in the strong dissipation limit
}

\author{
Tomasz Wasak $\odot,{ }^{1}$ Richard Schmidt, ${ }^{2}$ and Francesco Piazza ${ }^{1}$ \\ ${ }^{1}$ Max-Planck-Institut für Physik komplexer Systeme, Nöthnitzer Str. 38, 01187 Dresden, Germany \\ ${ }^{2}$ Max-Planck-Institut für Quantenoptik, Hans-Kopfermann-Str. 1, 85748 Garching, Germany
}

(Received 20 January 2020; accepted 24 December 2020; published 28 January 2021)

\begin{abstract}
The interplay between measurement and quantum correlations in many-body systems can lead to novel types of collective phenomena which are not accessible in isolated systems. In this work, we merge the Zeno paradigm of quantum measurement theory with the concept of polarons in condensed-matter physics. The resulting quantum-Zeno Fermi polaron is a quasiparticle which emerges for lossy impurities interacting with a quantum-degenerate bath of fermions. For loss rates of the order of the impurity-fermion binding energy, the quasiparticle is short lived. However, we show that in the strongly dissipative regime of large loss rates a long-lived polaron branch reemerges. This quantum-Zeno Fermi polaron originates from the nontrivial interplay between the Fermi surface and the surface of the momentum region forbidden by the quantum-Zeno projection. The situation we consider here is realized naturally for polaritonic impurities in charge-tunable semiconductors and can be also implemented using dressed atomic states in ultracold gases.
\end{abstract}

DOI: 10.1103/PhysRevResearch.3.013086

\section{INTRODUCTION}

The effect of measurement on the time evolution of a system is one of the most puzzling aspects of quantum dynamics $[1,2]$, making it drastically distinct from its classical counterpart. One paradigmatic example is the quantum-Zeno effect [3-5], whereby a frequent-enough measurement of a quantum system results in a localization of its state in Hilbert space [6]. Increasing the measurement rate from zero, the system dynamics first gains a nonunitary character due to the loss of information to the measurement device. However, above a threshold rate the trend is reversed: the dynamics tends asymptotically to a unitary limit within a Hilbert space reduced by the region that is forbidden due the measurement process.

Recently, growing attention is being devoted to the effect of repeated measurements on the dynamics of quantum many-body systems [7-13]. This has been motivated by experimental developments in controlling and selectively coupling complex quantum systems to their environment, as demonstrated with superconducting qubits [14-16], ultracold atoms [17-21], and molecules [22,23]. The interplay between measurement and intrinsic quantum correlations in many-body systems can lead to novel collective phenomena. Recently, two instances of such collective phenomena appearing in the quantum-Zeno limit have been predicted. First, analyzing the entanglement entropy, it was found that by

Published by the American Physical Society under the terms of the Creative Commons Attribution 4.0 International license. Further distribution of this work must maintain attribution to the author(s) and the published article's title, journal citation, and DOI. Open access publication funded by the Max Planck Society. increasing the measurement rate of a closed quantum manybody system, a phase transition from a volume-law entangled phase to a quantum Zeno phase with area-law entanglement can take place $[11,13]$. Second, a study of one-dimensional fermionic models with a localized dissipation (of Dirac delta type) revealed that the quantum Zeno projection is enhanced in the vicinity of the Fermi surface due to the existence of gapless modes [24].

In this work, we consider a situation where a strong dissipation acts on a subspace of the Hilbert space of an interacting quantum system, with the following question in mind: How are the many-body states affected by the emergence of a dissipation-induced constraint for the Hilbert space as the quantum-Zeno limit is approached? We focus on the paradigm of mobile quantum impurity problems at the border between few- and many-body physics and extend it to the regime of gain and loss of impurity particles. We demonstrate how the intrinsic correlation scales, governing the impurity problem, have a nontrivial interplay with the scale brought in by the dissipation-induced constraint, leading to qualitative modification of the many-body quantum states.

The concept of polarons plays a central role in condensedmatter physics, developed to understand the motion of an electron moving in a dielectric crystal [25-28]. The Fermi polaron, describing a mobile impurity interacting with a bath of fermions [29], is particularly important for the quantitative description of the physics of imbalanced Fermi mixtures [30,31] and sets the basis for our understanding of other strongly correlated systems [32-35]. The physics of Fermi polarons has become the center of significant interest due to recent experimental advances that allowed for its observation both with ultracold atoms [36-38] and more recently in charge-tunable monolayer semiconductors $[39,40]$.

So far, theoretical studies of the polaron paradigm are assuming a conserved number of impurities in a closed system. 
However, in addition to the fundamental interest in exploring the effect of strong measurement on quantum many-body systems in general, the recent experiments in charge-tunable monolayer semiconductors open a new frontier to study polarons in the presence of impurity loss and gain, as the corresponding rates can become comparable to the timescales of unitary dynamics [39]. In this work we develop a theoretical approach to describe such quantum driven-dissipative impurity dynamics. Using a diagrammatic, real-time Keldysh approach we show that, while for intermediate loss rates the polaron becomes expectedly short lived, in the quantum Zeno limit of large loss rates a long-lived polaron branch reemerges. This quantum-Zeno Fermi polaron is not simply a copy of the original polaron in absence of losses, but originates from the nontrivial interplay between the Fermi surface and the surface of the region forbidden by the quantum Zeno projection. This indeed leads to qualitative modifications of the dispersion of the impurity-fermion bound states as well as of the scattering continuum.

This complex interplay underlying the formation of the quantum-Zeno Fermi polaron requires a loss profile that can single out a nontrivial region in momentum space. This can be achieved by mixing the impurity with an additional lossy degree of freedom of much smaller mass. This is naturally the case for exciton-polariton impurities in charge-tunable monolayer semiconductors. It can also be engineered for ultracold atoms by coupling a stable electronic level to a short-lived level, and additionally using level-depending trapping potentials.

This paper is structured as follows. In Sec. II, we introduce a model describing the driven-dissipative Fermi polaron. In Sec. III, we discuss the results for the spectral response of the system. We show the emergence of the quantum-Zeno Fermi polaron for large loss rates and illustrate the interplay between the surface of the Zeno-forbidden region and the Fermi surface. In Sec. IV, we discuss in detail the concrete implementation of the Fermi polaron subject to strong dissipation. Finally, in Sec. V, we offer some concluding remarks and an outlook. Technical details are presented in the Appendices, where, in particular, we introduce a Keldysh diagrammatic approach to nonequilibrium Green's functions.

\section{DRIVEN-DISSIPATIVE IMPURITY IN A FERMI BATH}

We consider a system composed of a bath of fermions interacting with a vanishingly small density of impurity particles. The Hamiltonian of the system consists of three parts:

$$
\hat{H}=\hat{H}_{c}+\hat{H}_{f}+\hat{H}_{\mathrm{int}},
$$

where $\hat{H}_{c}=\sum_{\mathbf{k}} \varepsilon_{c}(\mathbf{k}) \hat{c}_{\mathbf{k}}^{\dagger} \hat{c}_{\mathbf{k}}$ and $\hat{H}_{f}=\sum_{\mathbf{k}} \varepsilon_{f}(\mathbf{k}) \hat{f}_{\mathbf{k}}^{\dagger} \hat{\mathbf{k}}_{\mathbf{k}}$ are freeparticle Hamiltonians with kinetic energies $\varepsilon_{c}(\mathbf{k})=\mathbf{k}^{2} / 2 m_{c}$ and $\varepsilon_{f}(\mathbf{k})=\mathbf{k}^{2} / 2 m_{f}$ of impurities and fermions, respectively. We assume that the interaction is a contact potential characterized by a coupling strength $U$, i.e.,

$$
\hat{H}_{\mathrm{int}}=U \int d \mathbf{r} \hat{c}^{\dagger}(\mathbf{r}) \hat{c}(\mathbf{r}) \hat{f}^{\dagger}(\mathbf{r}) \hat{f}(\mathbf{r}) .
$$

In this paper, all concrete calculations will be given for the two-dimensional case which has been realized in ultracold atoms $[38,41]$ and two-dimensional semiconductors [39,40].

Driven-dissipative impurities which are localized and immobile in a one-dimensional system have been recently studied in Refs. [42,43]. Here we consider mobile impurities which are subject to loss and thus require to be repumped into the system. To model the action of dissipation and pump, we employ the master equation

$$
\partial_{t} \hat{\varrho}(t)=-i[\hat{H}, \hat{\varrho}(t)]+\mathcal{L}_{d} \hat{\varrho}(t) .
$$

The dissipative Lindblad operator $\mathcal{L}_{d}$, acting on the density matrix of the whole system $\hat{\varrho}(t)$, splits into two parts, i.e., $\mathcal{L}_{d} \hat{\varrho}=\sum_{\mathbf{k}}\left\{\gamma(\mathbf{k}) D\left[\hat{c}_{\mathbf{k}}\right]+\Omega(\mathbf{k}) P\left[\hat{c}_{\mathbf{k}}\right]\right\} \hat{\varrho}$, which describe loss of particles with rate $\gamma(\mathbf{k})$ and their reinjection with rate $\Omega(\mathbf{k})$. The Markov form of the master equation (3) is valid as long as the reservoir of impurity particles has a correlation time much shorter than all other timescales characterizing the system [1]. We model here the dissipation strength by a momentumdependent profile, as it may be generated by a coupling of an impurity to a lossy degree of freedom, for example, excitons to microcavity photons or ground-state atoms to short-lived excited states.

We consider loss and injection of single impurities. These two incoherent processes are expressed by the superoperators $D[\hat{c}] \hat{\varrho} \equiv \hat{c}^{\dagger} \hat{\varrho} \hat{c}-\frac{1}{2}\left\{\hat{c}^{\dagger} \hat{c}, \hat{\varrho}\right\}$ and $P[\hat{c}] \equiv D[\hat{c}]+D\left[\hat{c}^{\dagger}\right]$. The action of each superoperator generates two terms. The anticommutator term corresponds to a non-Hermitian Hamiltonian evolution describing the damping and growth of the number of impurities, while the other term describes the noise which necessarily accompanies dissipation. In a stochastic quantum trajectory picture [1], the noise term generates projections of the wave function at random times induced by the measurement, which, in our case, corresponds to the detection of an impurity particle exiting or entering the system. In this picture, both the measurement and the damping (or growth) rate are set by $\gamma(\mathbf{k})$ [or $\Omega(\mathbf{k})$ ]. For this study, the specific form of the pump term $P$ is irrelevant since the excitation spectrum of the system does not depend on it as long as the average density of impurities is small.

In the limit of vanishing dissipation and drive, our model corresponds to the Fermi-polaron problem treated as a closed system. One approach to describe the polaronic states of an impurity coupled to a bath of fermions in this closed system is to employ a variational approach based on the so-called Chevy ansatz for the wave function [30,35,44-47]. In this ansatz the impurity motional degree of freedom is entangled with a single particle-hole excitation of the Fermi sea. The variation with respect to the expansion coefficients yields an equation that describes the properties of the lowest-lying polaron state, the so-called attractive polaron. Further variational methods, including higher-order particle-hole excitations, have also been developed to improve the Chevy expansion [31]. The variational approach [48] and a related non-self-consistent $T$-matrix approach [49] were used to capture the properties of an excited state of the polaron, known as the repulsive polaron. All these theoretical studies rely on the Hamiltonian description of the system and, therefore, are not directly applicable to the case of driven-dissipative impurities. 
In this work, we develop a diagrammatic approach based on nonequilibrium Green's functions (GFs) which extends the non-self-consistent $T$-matrix approach to driven-dissipative systems. We employ a path-integral formulation of our problem on the Keldysh real-time contour which allows for a straightforward inclusion of the Lindblad terms of the master equation [50,51]. Within this formalism, diagrammatic approaches are applicable in a way analogous to the equilibrium Matsubara formalism. In the following, we analyze the properties of the system in its steady state, where the GFs are time-translation invariant. The details of the calculations can be found in Appendix A.

In what follows, we will focus on the impurity limit where the number of impurities in the system is vanishingly small and the excitation spectrum of the system will not depend on the pump $\Omega(\mathbf{k})$ or the quantum statistics of impurities.

\section{FERMI POLARON WITH A LOSSY REGION IN IMPURITY-MOMENTUM SPACE}

\section{A. Impurity response function: Quantum-Zeno Fermi polaron}

As we shall see, the emergence of a quantum-Zeno Fermi polaron relies on impurity losses which single out at least one subset of momentum space. Such a subset we call the dissipative subspace (DS). In this section, we consider the simplest case of a loss function $\gamma(\mathbf{k})$ that is characterized by a single scale given by a cutoff momentum $k_{\gamma}$, and we assume that $\gamma(\mathbf{k})=\gamma_{0}$ if $|\mathbf{k}|<k_{\gamma}$ and is vanishing otherwise. Our approach is valid for any value of the loss rate $\gamma_{0}$. However, we will be interested in the case of large loss rates: $\gamma_{0} \gg E_{B}$ with $E_{B}$ the impurity-fermion binding energy. This leads to effective blocking of transitions between states involving the DS, which can be interpreted in terms of the quantum-Zeno effect. The exact form of the loss profile is not crucial for the physics described in what follows, and it is sufficient that the loss rate decays fast enough over the momentum scale $q_{B}=\sqrt{m_{f} E_{B}}$. We will demonstrate this by considering experimentally realistic cases in Sec. IV. Finally, we remark that the coupling strength $U$ is eliminated from the equations by finding the impurity-fermion binding energy $E_{B}$ (based on the poles of the vacuum $T$ matrix) in the limit of vanishing fermion density and with dissipation strength set to zero.

The spectral response of the system is encoded in the retarded impurity GF, which is given by

$$
G_{c}^{R}(\mathbf{k}, \omega)=\frac{1}{\omega-\varepsilon_{c}(\mathbf{k})+i \frac{\gamma(\mathbf{k})}{2}-\Sigma_{c}^{R}(p)+i 0},
$$

where the retarded self-energy $\Sigma_{c}^{R}(p)$ of the impurity contains information about the interaction with the surrounding bath of fermions; here and below the four-momentum $p \equiv(\mathbf{k}, \omega)$. The spectral function is then given by the following formula:

$$
\mathcal{A}_{c}(\mathbf{k}, \omega)=-2 \operatorname{Im}\left[G_{c}^{R}(\mathbf{k}, \omega)\right] .
$$

The spectral function satisfies the exact sum rule $\int \mathcal{A}_{c}(p) d \omega /$ $(2 \pi)=1$. It exhibits resonant peaks in correspondence to quasiparticle excitations with a width related to their inverse lifetime. In the absence of interactions with the fermions, the impurity spectral function $\mathcal{A}_{0, c}=-2 \operatorname{Im}\left[G_{0, c}^{R}\right]$ has resonance at its bare dispersion $\omega=\varepsilon_{c}(\mathbf{k})$, with a width determined by $\gamma(\mathbf{k})$. In presence of interactions, resonances appear at the renormalized frequencies $\omega=\varepsilon_{c}(\mathbf{k})-\operatorname{Re}\left[\Sigma_{c}^{R}(\mathbf{k}, \omega)\right]$. In this case, the inverse lifetime is set by the imaginary part of the pole position on the complex plane: $\operatorname{Im}[\omega(\mathbf{k})]$ satisfying $\left[G_{c}^{R}(\mathbf{k}, \omega(\mathbf{k}))\right]^{-1}=0$. For sufficiently large inverse lifetime the latter is approximately given by $\operatorname{Im}\left\{\left[G_{c}^{R}(\mathbf{k}, \omega)\right]^{-1}\right\}=$ $\operatorname{Im}\left[-\Sigma_{c}^{R}(\mathbf{k}, \omega)+i \gamma(\mathbf{k}) / 2\right]$.

An example of the impurity spectral function for gradually increasing $\gamma_{0}$ is presented in Fig. 1 for the mass balanced case. For small $\gamma_{0}$, two quasiparticle resonances are visible. The lower one in energy is the so-called attractive polaron. It corresponds to a state where the impurity attracts a cloud of surrounding fermions. The attractive polaron is long lived (the width of the peak vanishing for $|\mathbf{k}| \rightarrow 0$ ) and ceases to exist for large momenta, when the resonance enters the molecule-hole continuum. The latter appears as blurred region between the two polaron resonances. The second resonance is higher in energy and corresponds to a repulsive polaron state where the surrounding cloud is repelled by the impurity. For low enough losses and small enough momenta, the repulsive polaron has a shorter lifetime than the attractive polaron.

It is important to note that at any finite value of $\gamma_{0}$, while the losses affect bare impurity only within the DS defined by $|\mathbf{k}|<k_{\gamma}$, they do affect the polaron also outside the DS. The reason for this is that the polarons are impurities dressed by the interactions with the surrounding fermions and therefore involve a superposition of all the allowed momentum states. This statement is particularly explicit in the simple variational ansatz introduced at the end of Appendix A 2. Impurity losses thus affect the polarons in a nontrivial manner via the coupling between the impurity and the molecular state. The latter is very sensitive to dissipation. We note that for the value of $k_{\gamma}$ used in Fig. 1 and for momenta outside the DS, the repulsive polaron remains largely unaffected by the losses (see also Fig. 2). This is due to small coupling of the impurity with the bound state at high energies, where the repulsive polaron recovers the impurity-dispersion relation. The effect of losses outside the DS is most clearly visible in Figs. 1(c) and 1(d), where, for $\gamma_{0} \sim E_{B}$, the attractive-polaron peak is almost fully washed out.

Quite remarkably, for even larger losses $\gamma_{0} \gg E_{B}$, a resonance reappears below the repulsive polaron just outside the DS: $k>k_{\gamma}$. This resonance has a width which does not depend anymore on the loss rate $\gamma_{0}$ and is rather given only by the coupling to the molecule-hole continuum, as would be the case without losses. At the same time, the DS does not contain any spectral weight and is fully forbidden for the excitations. This phenomenology can be understood within the paradigm of the quantum-Zeno effect. Indeed, when the impurity occupies a state with $\mathbf{k} \in \mathrm{DS}$ it is lost immediately, which is equivalent to a frequent measurement performed within the DS. As a result, the dynamics of the particle is confined to the orthogonal subspace, i.e., $\mathbf{k} \notin \mathrm{DS}$. We note that for infinite dissipation strength, the equation for the pole of the impurity Green's function can be reproduced by a modification of the Chevy polaron ansatz for the wave function in which the momenta from DS are excluded.

The loss rate $\gamma_{0}$ does not appear explicitly anymore as the dynamics becomes unitary again in the quantum-Zeno limit $\gamma_{0} \gg E_{B}$ (see also Fig. 4 in the next section). Still, the size of 

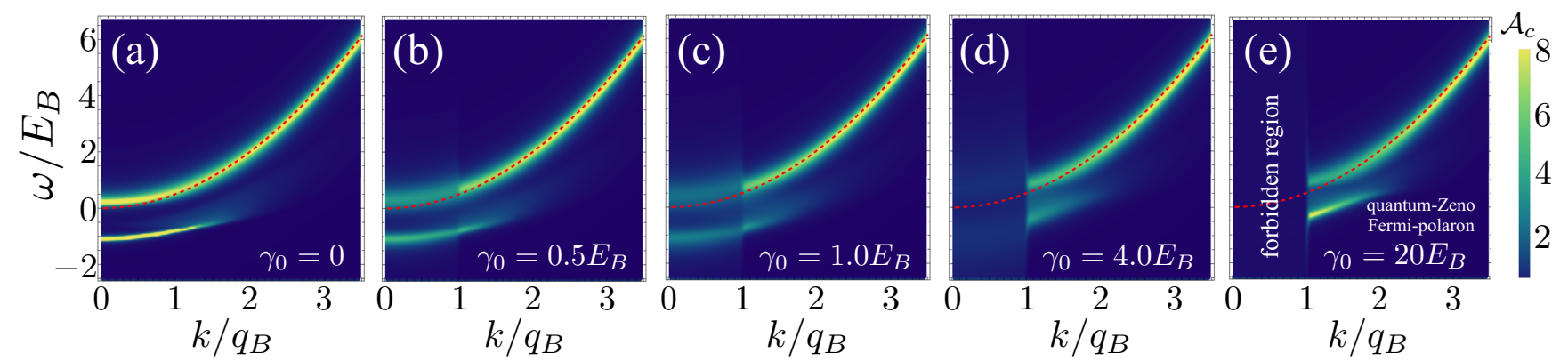

FIG. 1. The impurity spectral function $\mathcal{A}_{c}(k, \omega)$ (in the units of $\left.1 / E_{B}\right)$ for a two-dimensional (2D) system with the mass balance $m_{c}=m_{f}$. In (a)-(e) the strength of the loss is increased: $\gamma_{0} / E_{B}=0,0.5,1.0,4.0,20$. The energy is expressed in the units of the impurity-fermion binding energy $E_{B}$, and momenta are given in $q_{B}=\sqrt{m_{f} E_{B}}$. The Fermi energy $\epsilon_{F}=k_{F}^{2} / 2 m_{f}$ is set by the Fermi wave vector $k_{F}=0.7 q_{B}$. The color coding of the values for $\mathcal{A}_{c}$ is on the right. The cutoff momentum of the loss profile is at $k_{\gamma}=q_{B}$. The dashed red curve is the free dispersion relation $\omega=\varepsilon_{c}(k) \equiv k^{2} / 2 m_{c}$.

the DS $k_{\gamma}$ remains as further scale which plays a central role in the problem, as does the Fermi wave vector $k_{F}$. One effect of the nontrivial interplay between these scales is demonstrated in Fig. 2, where we show how the polaron dispersion depends on the size of the DS. By increasing $k_{\gamma}$ the attractive-polaron dispersion gradually changes from quadratic to linear. Moreover, the branch remains well defined up to larger values of $k$ compared to the lossless case and is also blueshifted. This is evident from Fig. 2(d) where the resonance appears also for $\omega>0$. In addition, the spectral weight of the repulsive polaron is transferred to the attractive branch. By increasing $k_{\gamma}$ the attractive polaron peak becomes broader due to the entrance into the molecule-hole continuum.

\section{B. Molecular response function: Quantum-Zeno scattering}

In the interaction between an impurity and a fermion, the formation of paired (or molecular) states plays a crucial role. Our approach based on an auxiliary molecular field allows to rewrite the impurity self-energy in the following way, see Appendix A:

$$
\Sigma_{c}^{R}(\mathbf{k}, \omega)=\frac{1}{V} \sum_{\mathbf{k}^{\prime} \in \mathrm{FS}} G_{\Delta}^{R}\left(\mathbf{k}+\mathbf{k}^{\prime}, \omega+\varepsilon_{f}\left(\mathbf{k}^{\prime}\right)\right),
$$

from which we see that the renormalization of the impurity is fully determined by the molecular GF. The molecular GF is at the same time directly related to the $T$ matrix describing the scattering between the impurity and a fermion. Specifically, we will define $T(\mathbf{k}, \omega)=G_{\Delta}^{R}(\mathbf{k}, \omega)$, where $\mathbf{k}$ is the momentum of the molecule and $\omega$ is its energy. In Appendix A 2 we calculate the molecular self-energy in the impurity limit, which takes the form

$$
\Sigma_{\Delta}^{R}(\mathbf{k}, \omega)=\frac{1}{V} \sum_{\mathbf{k}^{\prime} \notin \mathrm{FS}} \frac{1}{\omega-\varepsilon_{c}\left(\mathbf{k}-\mathbf{k}^{\prime}\right)-\varepsilon_{f}\left(\mathbf{k}^{\prime}\right)+i \frac{\gamma\left(\mathbf{k}-\mathbf{k}^{\prime}\right)}{2}+i 0} .
$$

This object then determines the molecular GF, which reads as

$$
G_{\Delta}^{R}(p)=\frac{1}{-v^{2}-\Sigma_{\Delta}^{R}(p)+i 0},
$$

where $v^{2}=-1 / U$.

In the case of a single fermion interacting with a lossless impurity, which is recovered by sending both $\epsilon_{F}$ and $\gamma_{0} \rightarrow 0$, we obtain (in two-spatial dimensions) the usual form of the vacuum $T$ matrix:

$$
T^{-1}(\mathbf{k}, \omega)=-\frac{m_{r}}{2 \pi}\left[\ln \left(\frac{E+i 0}{E_{B}}\right)-i \pi\right] \equiv T_{0}^{-1}(E),
$$

which depends only on the kinetic energy of the relative motion of the particles $E=\omega-k^{2} / 2 M$ with $M=m_{c}+m_{f}$ the total mass; the index " 0 " in $T_{0}$ refers to the vacuum case without losses. The $T$ matrix has a pole at $E=-E_{B}$ signaling the presence of a bound state at that energy.
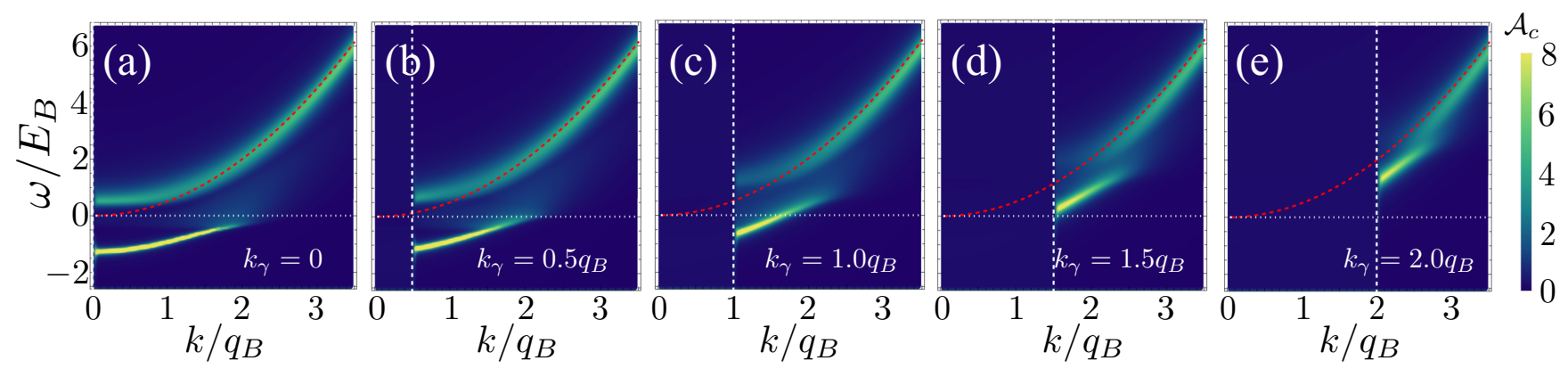

FIG. 2. Impurity spectral function $\mathcal{A}_{c}(\mathbf{k}, \omega)$ (in units of $E_{B}^{-1}$ ) as a function of $|\mathbf{k}|$ and $\omega$ (in units of $q_{B}$ and $E_{B}$, respectively) for different sizes of the dissipative subspace: $k_{\gamma} / q_{B}=0,0.5,1.0,1.5,2.0$ [from (a) to (e)]. The Fermi energy $\epsilon_{F}=k_{F}^{2} / 2 m_{f}$ is set by $k_{F}=q_{B}$, and $\gamma_{0}=20 E_{B}$. The dotted horizontal line depicts $\omega=0$. The dashed vertical line [in (b)-(e)] displays $k_{\gamma}$. The dashed red parabolic curve shows the dispersion relation $\omega=k^{2} / 2 m_{c}$ of the free impurity. 


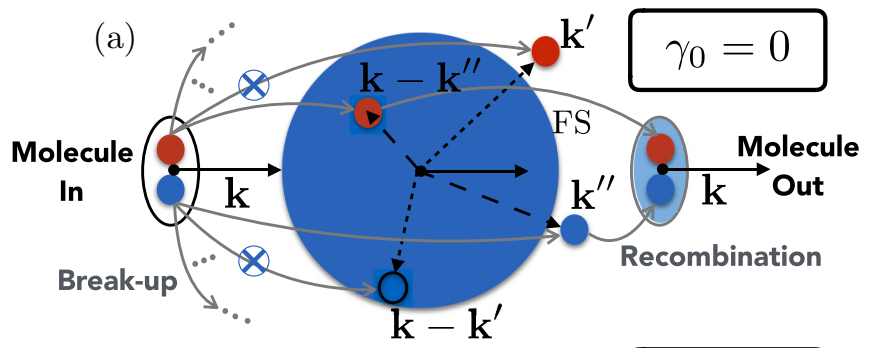

(b)

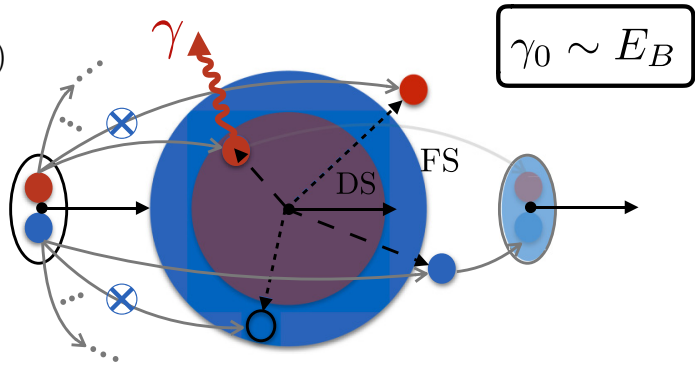

(c)
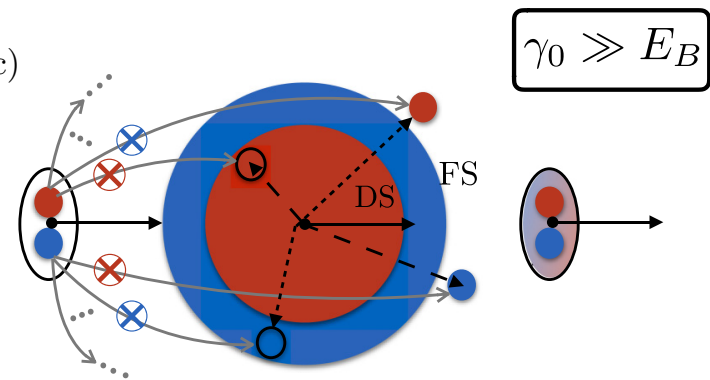

FIG. 3. The polaron is formed because the impurity (red circle) forms a molecular state with a fermion (blue circle). Crucially, the dynamics of molecules is in turn generated by virtual breakup processes followed by recombination, as illustrated here pictorially to complement the diagrammatic representation of Fig. 8(b) and the formal expression of Eq. (A9) for the molecular self-energy. A molecule with momentum $\mathbf{k}$ temporarily breaks up into an impurity with momentum $\mathbf{k}^{\prime}$ and a fermion with momentum $\mathbf{k}-\mathbf{k}^{\prime}$. The total effect is obtained by summing over all processes involving different momenta, as graphically indicated by the dots. In (a) the dissipationless case is considered, where the Pauli principle forbids processes creating a fermion within the Fermi surface (FS). In (b), moderate dissipation at a rate $\gamma_{0}$ comparable with the impurity-fermion binding energy $E_{B}$ is added within the DS, which effectively makes the molecular state dissipative as well. Finally, in (c) the strongly dissipative regime is considered, where the quantum-Zeno effect forbids the creation of an impurity within the DS.

For $\epsilon_{F}>0$, the scattering properties are modified due to the presence of the fermionic medium. Here, an important role is played by the Pauli exclusion principle, which results in the constraint $\mathbf{k}^{\prime} \notin \mathrm{FS}$ on the summation variable in $\Sigma_{\Delta}^{R}$ [see Eq. (7)]. This restricts the number of scattering processes which modify the molecule propagation. As already pointed out, these processes are the only source of the dynamics of the molecular state since the only frequency-momentum dependence of the molecule GF from Eq. (8) is induced by the self-energy $\Sigma_{\Delta}^{R}$. As shown pictorially in Fig. 3, and diagrammatically in Fig. 8(b), the molecule dynamics receives a contribution from all energy-and-momentum-conserving processes where a molecular state temporarily breaks up into an impurity plus a fermion. In absence of losses, corresponding
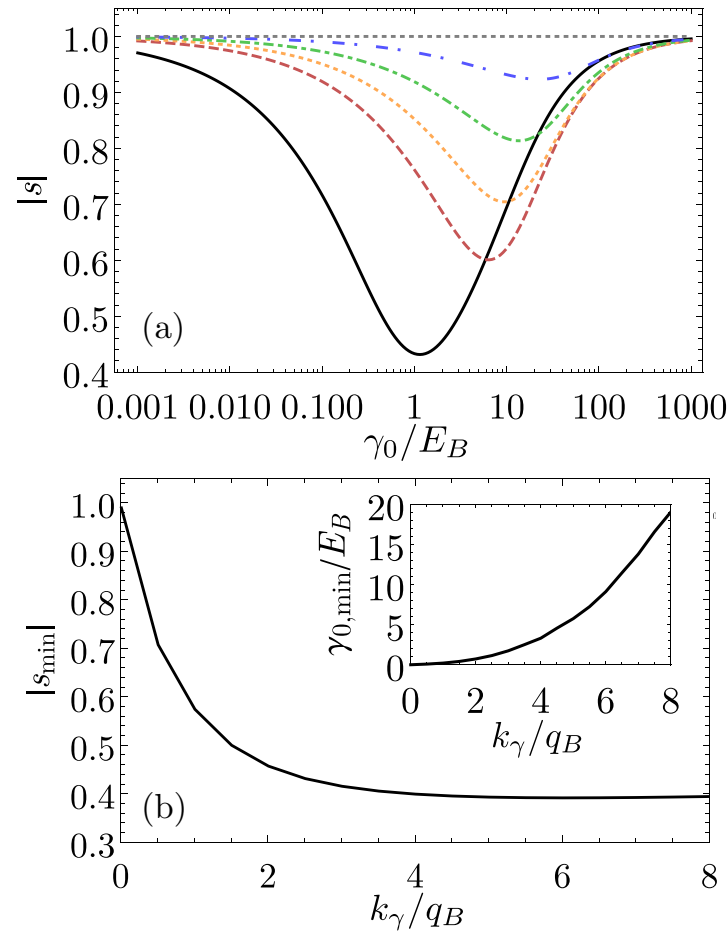

FIG. 4. (a) The $S$ matrix $|s(\mathbf{k}, \omega)|=\left|e^{i \delta(\mathbf{k}, \omega)}\right|$ as a function of the loss rate $\gamma_{0}$ (in units of $E_{B}$ ), computed at threshold $\omega=\omega_{\text {th }}$ for $k / q_{B}=0.05$ (solid black), 0.5 (dashed red), 1 (orange dotted), 2 (dotted-dashed green), 5 (double-dotted-dashed blue); here $k_{\gamma}=$ $2.5 q_{B}$. The dotted gray horizontal line is for infinitely fast losses [see Eq. (11)]. (b) The minimum of $\left|s_{\min }\right| \equiv \min _{\gamma_{0}}\left|s\left(\gamma_{0}\right)\right|$ as a function of $k_{\gamma}$ calculated for $k=0.05 q_{B}$. The inset of (b) shows the value of $\gamma_{0}$ for which $|s|$ takes the minimum value, denoted by $\gamma_{0, \min }$. In both panels, $m_{c}=m_{f}$.

to Fig. 3(a), all processes creating a fermion within the FS are forbidden by Pauli blocking. This case has been already studied in the literature for the mass-balanced case [52-54], and the self-energy can be computed analytically [49]. The result can be generalized to the mass-imbalanced case [55], but we do not present it here.

We now turn to the situation where impurity losses are present in the DS. The relevant processes in this case are shown in Fig. 3(b): whenever the molecule breaks up creating an impurity within the DS, there is a finite probability for the impurity to be lost so that the molecule cannot be rebuilt. Considering the sum over all processes, this induces dissipation for the dressed molecular states. From Eq. (7) we see that for momenta $\mathbf{k}^{\prime}$ satisfying $\mathbf{k}-\mathbf{k}^{\prime} \in \mathrm{DS}$, the contribution to $\Sigma_{\Delta}^{R}$ is negligible if the loss rate is very large: $\gamma_{0} \gg E_{B}$. If the number of fermions is vanishing, performing a shift in $\mathbf{k}^{\prime}$ we can rewrite the sum as

$$
\left.\Sigma_{\Delta}^{R}(\mathbf{k}, \omega)\right|_{\epsilon_{F}=0=\frac{1}{\gamma_{0}}}=\frac{1}{V} \sum_{\mathbf{k}^{\prime \prime} \notin \mathrm{DS}} \frac{1}{\omega-\varepsilon_{f}\left(\mathbf{k}-\mathbf{k}^{\prime \prime}\right)-\varepsilon_{c}\left(\mathbf{k}^{\prime \prime}\right)+i 0} .
$$

Comparing Eq. (7) for $\gamma=0$ with Eq. (10), we see that the dissipative subspace in the limit of large losses plays a role analogous to a Fermi surface, as illustrated in Fig. 3(c). In other words, at the level of scattering physics the forbidden DS 
in momentum space results from an effective "Pauli blocking" emerging in the quantum-Zeno limit [17].

The analogy between the forbidden DS and the FS is, however, not complete. For example, one can excite fermions out of the FS but not the DS. As for the analogous case of a FS, the sum in Eq. (10) can be evaluated analytically in presence of the forbidden DS, leading to

$$
\begin{aligned}
\left.T(\mathbf{k}, \omega)\right|_{\epsilon_{F}=0=\frac{1}{\gamma_{0}}}= & T_{0}\left[\frac{z}{2}-\left(\frac{1}{2}-\frac{m_{r}}{m_{f}}\right) \varepsilon_{f}(\mathbf{k})\right. \\
& \pm \frac{1}{2} \sqrt{\left.\left[z-\varepsilon_{f}(\mathbf{k})\right]^{2}-4 \varepsilon_{f}(\mathbf{k}) \frac{k_{\gamma}^{2}}{2 m_{f}}\right]}
\end{aligned}
$$

where $z=\omega-\frac{k_{\gamma}^{2}}{2 m_{r}}+i 0$, and $\pm=\operatorname{sign}\left\{\operatorname{Re}\left[z-\varepsilon_{f}(\mathbf{k})\right]\right\}$. Interestingly, in this case the $T$ matrix can be expressed in terms of the vacuum two-body $T_{0}$ matrix. This is a property of the two-dimensional (2D) geometry [49] and does not occur in other dimensions.

In order for the quantum-Zeno Fermi polaron to appear, the existence of both a FS and a forbidden (or at least strongly dissipative) DS is required. In this case the molecular selfenergy can be rewritten as

$$
\left.\Sigma_{\Delta}^{R}(\mathbf{k}, \omega)\right|_{\frac{E_{B}}{\gamma_{0}} \simeq 0} \simeq \frac{1}{V} \sum_{\substack{\mathbf{k}^{\prime} \notin \mathrm{FS} \\ b k^{\prime \prime} \notin \mathrm{DS}}} \frac{\delta_{\mathbf{k}^{\prime \prime}+\mathbf{k}^{\prime}, \mathbf{k}}}{\omega-\varepsilon_{c}\left(\mathbf{k}^{\prime \prime}\right)-\varepsilon_{f}\left(\mathbf{k}^{\prime}\right)+i 0},
$$

where the sum over all processes is subject to two constraints.

An important feature of the quantum-Zeno limit is that the emergence of a forbidden subspace coincides with the reemergence of unitary dynamics. This becomes evident when evaluating the scattering $S$ matrix. In closed-system dynamics, the $S$ matrix conserves the total probability as expressed by the unitarity condition $S^{\dagger} S=\mathbb{1}$, where $\mathbb{1}$ is the operator identity. In the presence of dissipation, we have instead a decrease of the probability for some of the scattering channels and consequently $\left|e^{i \delta}\right|<1$, where the phase shift $\delta(E)$ characterizes the eigenvalue $s=e^{i \delta}$ of the scattering $S$ matrix at a collision energy $E=E_{\text {rel }}=k^{2} / 2 m_{r}$. In the quantum-Zeno limit, we thus expect $|s| \rightarrow 1$ for $\gamma_{0} \rightarrow \infty$.

To proceed further, we relate $s$ to the $T$ matrix. For twobody collisions in 2D without dissipation, the on-shell $T$ matrix is related to the $S$ matrix by $[54,56]$

$$
s_{0}(\mathbf{k}, \omega)=1+\frac{i t_{0}(\mathbf{k}, \omega)}{2},
$$

where for our case of $s$-wave collisions, $t_{0}=-2 m_{r} T_{0} ; \mathbf{k}$ is the momentum of the center of mass and $\omega=E_{\mathrm{rel}}+k^{2} / 2 M$ is the total energy, i.e., the sum of the kinetic energy of the center of mass and the collision energy of relative motion $E_{\mathrm{rel}}$. This relation implies $s_{0}=e^{i \delta_{0}}$ and in particular $\left|s_{0}\right|^{2}=1$ for the scattering states with $E_{\text {rel }}>0$, as required by unitarity.

In our open system, we generalize the relation (13) to the case where one of the two scattering partners is subject to losses (this is different, but related to the case of inelastic collisions [57]):

$$
s(\mathbf{k}, \omega)=1+\frac{i t(\mathbf{k}, \omega)}{2},
$$

where $t=-2 m_{r} T$ and $\omega=k^{2} / 2 M+E_{\mathrm{rel}}$. This leads to $|s|=1$ for collisions in vacuum or in medium (without dissipation) while $|s|<1$ for nonzero loss rate $\gamma_{0}$. These relations are satisfied provided that in all these cases the scattering states exist, that is, the states are in the continuum $E_{\text {rel }}>0$ and the particles can move away to infinity (we are not considering here cases where scattering states might not be well defined due to the form of the scattering potential itself). The second condition means that the momentum of the initial or final state should not be subject to loss from the DS. For an impurity moving with momentum $\mathbf{k}_{c}=\frac{m_{c}}{M} \mathbf{k}+\mathbf{k}_{\text {rel }}$, where $\mathbf{k}$ is the total momentum of the impurity-fermion system and $\mathbf{k}_{\mathrm{rel}}$ is the momentum of the relative motion, the condition $\mathbf{k}_{c} \notin \mathrm{DS}$ implies that the particles can escape after the collision for all directions of $\mathbf{k}_{\mathrm{rel}}$, if

$$
\left(\sqrt{2 m_{r} E_{\mathrm{rel}}}-\frac{m_{c}}{M} k\right)^{2}>k_{\gamma}^{2} .
$$

In order to simplify the analysis below, we set the threshold value to $E_{\text {rel }}^{\text {th }} \equiv \omega_{\text {th }}-k^{2} / 2 M=\frac{\left[\left(1-\frac{m_{r}}{m_{c}}\right) k+k_{\gamma}\right]^{2}}{2 m_{r}}$. We note that, while for for all collision energies $E_{\text {rel }}>E_{\text {rel }}^{\text {th }}$ the condition (15) is satisfied, there might also be conditions for which it is satisfied in a window of energies $0<E_{\text {rel }}<E_{\text {rel }}^{\text {th }}$.

In Fig. 4(a) we show $|s|$ computed for the energy $E_{\text {rel }}^{\text {th }}$ as a function of the loss rate $\gamma_{0}$ for various total momenta of the particles $k$ ranging from 0.05 to $5.0 q_{B}$. We observe that for small values of $\gamma_{0} \ll E_{B}$, the low momenta $k \ll k_{F}$ are affected by the loss, reducing the value of $|s|$ below unity. Increasing the loss rate leads to a further decrease of $|s|$, as the scattering becomes dominated by the presence of DS. For low momenta, the deviation from unitarity becomes maximal when $\gamma_{0}$ is on the order of $E_{B}$. For still higher $\gamma_{0}$, the value of $|s|$ increases towards unitarity, as the contributions to $s$ from DS become negligible. In the limit of very large $\gamma_{0}$ we observe $|s| \rightarrow 1$. The deviations from unitarity are decreasing with increasing the scattering momentum. We observe that for a fixed value of $k$, the threshold value of $\gamma_{0}$ (called $\left.\gamma_{0, \min }\right)$, beyond which $|s|$ starts to increase (called $\left|s_{\min }\right|$ ), is on the order of $E_{B}$. In Fig. 4(b), we display the maximum violation of unitarity (main panel) and the threshold value $\gamma_{0}$ (inset), as a function of the extent of DS $k_{\gamma}$. We see that the larger the DS becomes, the deeper the minimal value of $|s|$ is, which saturates for $k_{\gamma} \rightarrow \infty$ (see the main panel). The significant deviations of the unitarity are to be expected when $k_{\gamma} \gtrsim q_{B}$ and $\gamma_{0} \gtrsim E_{B}$. The increase of the DS leads to a fast increase of the threshold value of $\gamma_{0 \text {,min }}$. Comparing this to Fig. 4(a), we see that to reach the Zeno limit when $|s| \approx 1$ requires an order of magnitude of increase of $\gamma_{0}$ as compared to $\gamma_{0, \min }$. We note that in this figure, $k_{F}=0$ and the value of $\omega$ is set to its limiting case $\omega_{\text {th }}$. For larger $\omega$ the value of $|s|$ increases, and similarly $|s|$ grows with $k_{F}$.

A further characterization of the scattering can be obtained by analyzing the phase of the $T$ matrix. We compute $\operatorname{Arg}[-T(\mathbf{k}, \omega)]$ for fixed $\mathbf{k}$ as a function of $\delta \omega$, where we define $\omega=k^{2} / 2 M+\delta \omega$. In Fig. 5 we consider the vacuum case at $k=0$. In absence of losses [dotted red lines in Figs. 5(a) and 5(b)], the phase experiences a $\pi$ jump when crossing the bound state. Here, the phase is zero for $\delta \omega<-E_{B}$, and is $\pi$ for $-E_{B}<\delta \omega<0$, and for $\delta \omega>0$ it decays monotonically. 

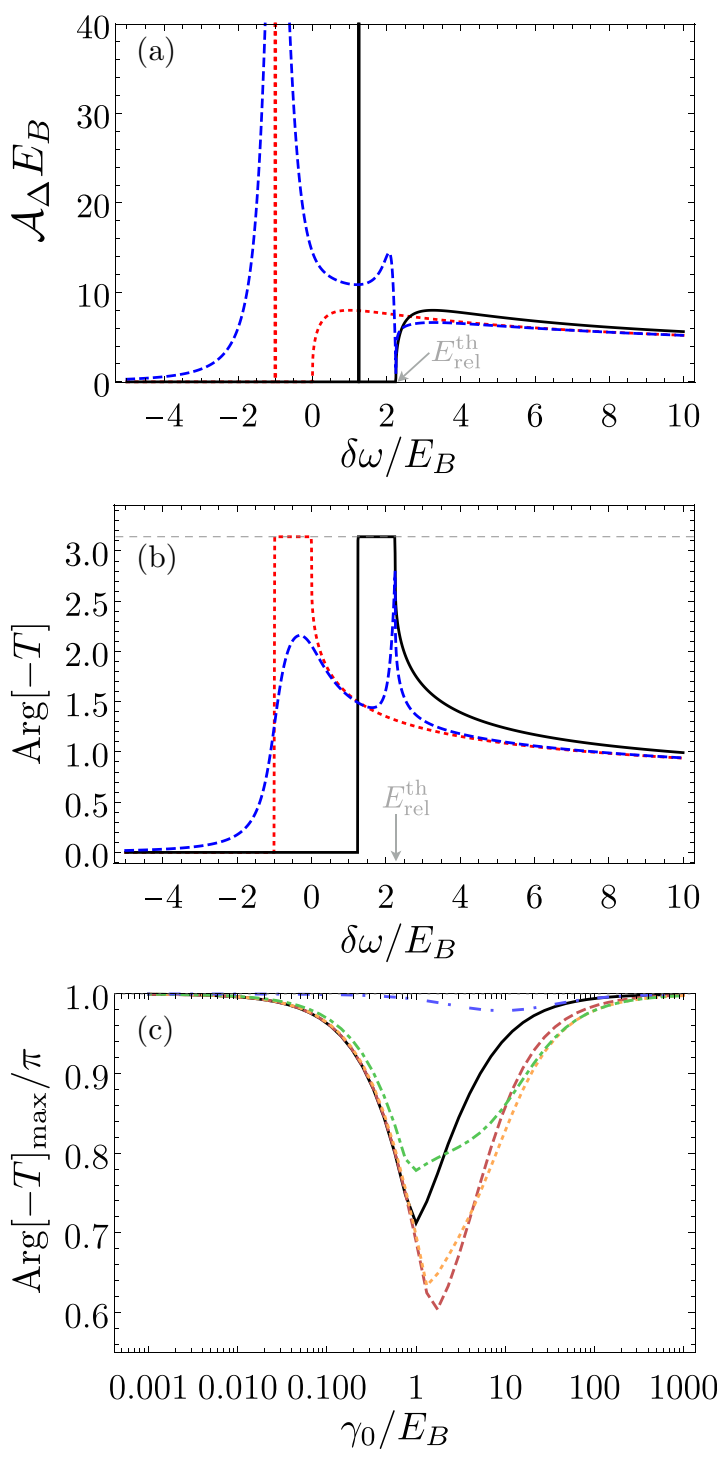

FIG. 5. (a) Spectral function of the molecule $\mathcal{A}_{\Delta}=$ $-2 \operatorname{Im}[T(\mathbf{k}, \omega)]$ calculated for $k=0$ and $\omega=k^{2} / 2 M+\delta \omega$; here $k_{F}=0, k_{\gamma}=1.5 q_{B}$. The dotted red line: the vacuum, lossless case [cf. Eq. (9)]. The dashed blue line: $\mathcal{A}$ in the presence of moderate loss $\left(\gamma_{0}=E_{B}\right)$. The black line: infinite loss case [cf. Eq. (11)]. The arrow indicates the threshold $E_{\mathrm{rel}}^{\mathrm{th}}=2.25 E_{B}$. (b) The phase of the $T$ matrix, i.e., $\operatorname{Arg}[-T]$. The color code and parameters are as in (a). (c) The maximum value of $\operatorname{Arg}[-T]$, calculated with respect to parameter $\delta \omega$, as a function of $\gamma_{0}$. The color coding is the same as in Fig. 4(a). In all the panels, $m_{c}=m_{f}$.

In presence of losses, as discussed above, the scattering threshold is shifted by $k_{\gamma}^{2}$ since impurities can escape only for momenta outside the DS. Therefore, the phase of the $T$ matrix is analytical and the scattering phase shift defined only for $\omega>k_{\gamma}^{2}$ [see blue dashed and solid black lines in Figs. 5(a) and 5(b)].

For finite loss rates (blue dashed lines), the jump in the $T$-matrix phase has a local maximum at the bound state but does not reach the maximal value $\pi$. By further increasing the energy the $T$-matrix phase then grows again to reach $\pi$ at the threshold value and then decreases monotonically and faster than in the lossless case above threshold.
The $T$-matrix phase behaves nontrivially upon increasing the loss rate. The double-peak structure present below threshold develops, in the quantum-Zeno limit of infinite loss rate, into a single nonanalytical plateau identical to the one present in the lossless case, apart from a blueshift of the threshold and the bound state. As we argued above, the vacuum scattering in the quantum-Zeno limit is the same as the lossless scattering in presence of a Fermi surface, where the role of $k_{F}$ is played by $k_{\gamma}$. Consistently with this, the phase of the $T$ matrix in the quantum-Zeno limit and in particular the scattering phase shift indeed behaves as its lossless counterpart in the presence of a medium, which has been studied in [53,54,58].

In particular, one can quantify the distance from the quantum-Zeno limit by the size of the jump of the $T$-matrix phase across the bound state. This is shown in Fig. 5(c), where we plot $\operatorname{Arg}[-T(\mathbf{k}, \omega)]$ maximized over $\delta \omega$. We see that for increasing $\gamma_{0}$, the maximum jump of the phase of $T$ drops, and for $\gamma_{0} \sim E_{B}$ it reaches a minimum, after which the further increase of $\gamma_{0}$ leads to recovery of the maximal jump of the phase.

The recovery of unitary scattering dynamics explains the reappearance of an attractive-polaron branch for large loss rates shown in Fig. 1. As already discussed in Sec. III A, this quantum-Zeno polaron features a clearly different dispersion affected by the presence of the additional scale $k_{\gamma}$, as demonstrated by Fig. 2 .

We now turn to the discussion of the interplay between $k_{\gamma}$ and the Fermi scale $k_{F}$, which is best understood by analyzing the molecular spectral function. In Fig. 6 we show the spectral function $\mathcal{A}_{\Delta}$ for various values of $k_{\gamma}$ at a fixed density of fermions $k_{F}=q_{B}$. Moreover, we choose a finite but large loss rate $\gamma_{0}=20 E_{B}$, where the scattering is already close to the quantum-Zeno regime.

For $k_{\gamma}=0$, i.e., without losses [see Fig. 6(a)], the spectrum consists of a molecular bound state (in this dissipationless case, we artificially set a finite lifetime for the bound state so that it is visible in the plot) and a continuum. In the nonself-consistent $T$-matrix approximation employed here, the boundaries of the continuum can simply be understood using energy and momentum conservation in the processes of Fig. 3 . This has been discussed already in previous works [49,54], but we reproduce the arguments here since we will extend them below to the quantum-Zeno limit. A molecule with energy $\omega$ and momentum $|\mathbf{k}|=k$ can decay into the impurity and a fermion. The conservation laws require $\omega=\varepsilon_{f}\left(\mathbf{k}^{\prime}\right)+\varepsilon_{c}\left(\mathbf{k}^{\prime \prime}\right)$ and $\mathbf{k}=\mathbf{k}^{\prime}+\mathbf{k}^{\prime \prime}$. The Pauli blocking sets an additional constraint $\left|\mathbf{k}^{\prime}\right|>k_{F}$. For example, setting $k=0$, we have $\mathbf{k}^{\prime}=$ $-\mathbf{k}^{\prime \prime}$ and the energy conservation leads to $\omega=\left|\mathbf{k}^{\prime \prime}\right|^{2} / 2 m_{r} \leqslant$ $k_{F}^{2} / 2 m_{r}$. Therefore, for $\omega \geqslant k_{F}^{2} / 2 m_{r}$ the molecule can decay into an impurity-fermion pair. Moreover, one can show that for $k \approx k_{F}$, the threshold for the molecular decay behaves as $k_{F}^{2} / 2 m_{f}+\left(k-k_{F}\right)^{2} / 2 m_{c}$. In general, for $k<m_{c} k_{F} / m_{r}$, the continuum boundary has the form $\left(k_{F}-m_{r} k / m_{c}\right)^{2} / 2 m_{r}+$ $k^{2} / 2 M$, and for $k>m_{c} k_{F} / m_{r}$ it is $k^{2} / 2 M$, where $M=m_{c}+$ $m_{f}$ is the total mass. Consequently, for $k_{\gamma}=0$, the continuum has a minimum at $k=k_{F}$ and $\omega=\epsilon_{F}$. For smaller $k<k_{F}$, it is blueshifted due to Pauli blocking, and for $k=0$ it starts at $\omega=k_{F}^{2} / 2 m_{r}$.

For a finite size of the DS and in the quantum-Zeno limit, several new features emerge in the molecular spectrum with 


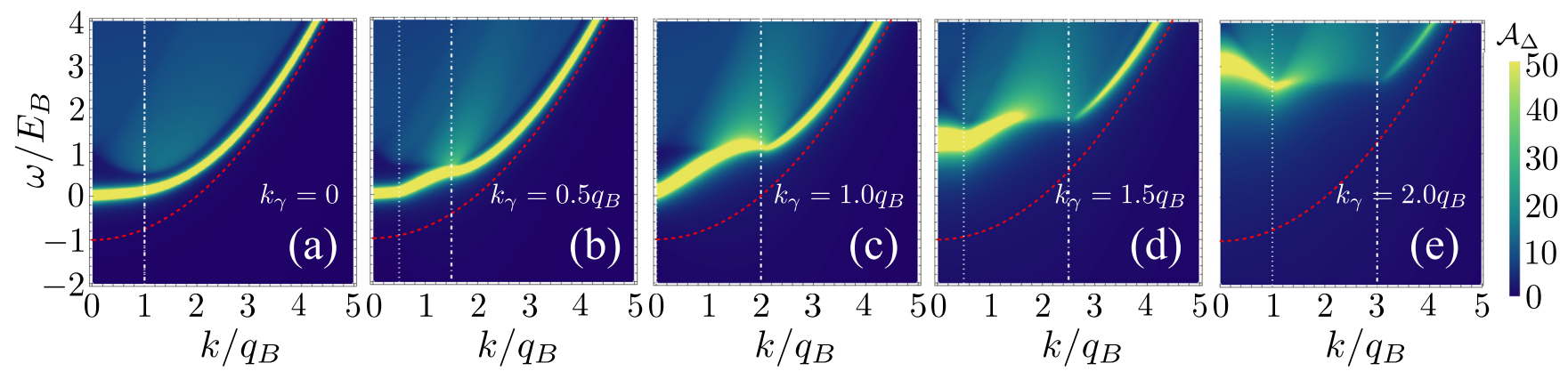

FIG. 6. Molecular spectral function $\mathcal{A}_{\Delta}(\mathbf{k}, \omega)$ (in units of $q_{B}^{2} E_{B}$ ) for $m_{c}=m_{f}$ as a function of $|\mathbf{k}|$ (in units of $q_{B}$ ) and $\omega$ (in units of $E_{B}$ ) for different sizes of the dissipative subspace: $k_{\gamma} / q_{B}=0,0.5,1.0,1.5,2.0$ [from (a) to (e)]. The Fermi energy $\epsilon_{F}=k_{F}^{2} / 2 m_{f}$ is set to $k_{F}=q_{B}$, and $\gamma_{0}=20 E_{B}$. The dotted and dotted-dashed vertical lines indicate $\left|k_{\gamma} \mp k_{F}\right|$. The dashed red curve is the molecular free dispersion given by $\omega=-E_{B}+k^{2} / 2 M$.

respect to the lossless case. For small $k_{\gamma}$, the spectrum is affected mostly in the vicinity of the Fermi momentum, namely, in the region $k_{F}-k_{\gamma}<k<k_{F}+k_{\gamma}$, as can be seen from Figs. 6(b) and 6(c). By increasing $k_{\gamma}$, the spectral weight of the continuum transfers to $\omega \geqslant k_{\gamma}^{2} / 2 m_{c}+k_{F}^{2} / 2 m_{f}$ in the region $k \approx k_{F}$. Due to the presence of the DS, for smaller $k$ than $\left|k_{F}-k_{\gamma}\right|$ the spectrum is blueshifted more rapidly, and at $k=0$ it reaches $Q^{2} / 2 m_{r}$, where $Q=\max \left(k_{F}, k_{\gamma}\right)$. Notice that, for $k$ lying in the interval $k_{F}-k_{\gamma}<k<k_{F}+k_{\gamma}$, the bound-state dispersion is linear in $k$. For even larger $k_{\gamma}$, the effect of the Pauli blocking is enhanced by the presence of the DS, and the bound-state energy is blueshifted even more. With increasing $k$ the bound-state energy drops, and enters the continuum for larger $k$. Due to the forbidden regions in the formation of a molecule, the impurity and the fermion must have momenta not smaller than $k_{\gamma}$ and $k_{F}$, respectively. Therefore, the molecule with the smallest energy and momentum will have $k=\left|k_{F}-k_{\gamma}\right|$. Since the continuum starts now at $k_{\gamma}^{2} / 2 m_{c}+k_{F}^{2} / 2 m_{f}$, the molecular state can appear at this energy also for $k=k_{\gamma}+k_{F}$. This gives rise to the appearance of two minima in the bound-state dispersion visible in Fig. 6(e). This analysis makes apparent the crucial role played by the size of the DS in the quantum-Zeno regime, where the molecule and, in turn, the polaron dispersion can be modified qualitatively.

The results presented in this section combined with Figs. 1 and 2 highlight an important ingredient for the appearance of strong qualitative effects of dissipation on molecular and polaron states. It is namely the molecular bound state which is strongly modified by the losses, while the continuum experiences weaker modifications. This is reflected by the behavior of the polarons: only the attractive polaron is strongly affected by loss. This leads us to the expectation that the phenomenology we propose here will crucially depend on the presence or absence of the molecular bound state and therefore on the dimensionality.

Finally, we end this discussion by noting that all the results are obtained within the non-self-consistent $T$-matrix approach, whose limitations are known [49]. The full solution would require to employ self-consistency of the Green's functions and would lead to modification of the molecular spectral function shifting the position of the molecular and polaron resonances and affecting their lifetimes. These corrections, however, in the configurations and regimes considered affect the quantities of present interest only quantitatively and do not change the overall physical picture.

\section{IMPLEMENTATION OF THE MODEL}

In this section, we discuss how the physics discussed so far could be realized in experiment. Starting from a setup featuring a density-density interaction between fermions and impurity particles, the goal is to realize momentum-dependent losses for the impurities. The loss profile needs to single out a given region of momentum space by being much larger there than elsewhere.

Recently, it was demonstrated that it is possible to explore the two-dimensional Fermi-polaron problem in transition metal dichalcogenide monolayer semiconductor embedded into microcavities $[39,40]$. In this type of setup, excitons are deeply bound and hybridize with the cavity photons, forming polaritons. By optically exciting a small number of polaritons it is possible to create impurities that feature a mass that is much smaller than that of the surrounding electrons since photons propagate much faster. More important for the present discussion is the fact that the photons feature roughly momentum-independent losses due to mirror leakage. These are inherited by the polaritons in a momentum-dependent fashion, as the photonic admixture indeed depends on momentum. This means that for one of the two polaritons the loss rate will decrease by increasing momentum, while the opposite is true for the other polariton.

The simplest model for the above scenario is given by the following Hamiltonian:

$$
\begin{gathered}
H_{\mathrm{eff}}=\sum_{\mathbf{k}}\left\{\epsilon_{c}(\mathbf{k}) \hat{c}_{\mathbf{k}}^{\dagger} \hat{c}_{\mathbf{k}}+\left[-\delta+\epsilon_{d}(\mathbf{k})\right] \hat{d}_{\mathbf{k}}^{\dagger} \hat{d}_{\mathbf{k}}\right. \\
\left.+\Omega_{R}\left(\hat{c}_{\mathbf{k}}^{\dagger} \hat{d}_{\mathbf{k}}+\hat{d}_{\mathbf{k}}^{\dagger} \hat{c}_{\mathbf{k}}\right)\right\},
\end{gathered}
$$

where $\epsilon_{c, d}(\mathbf{k})$ is the dispersion of the exciton and the photon, respectively, and $\delta$ the relative energy offset. $\Omega_{R}$ is the Rabi frequency of interconversion between exciton and photon. The latter is subject to losses at a rate $\gamma_{d}$, corresponding to the Lindblad operator

$$
\mathcal{L}_{\text {diss }}=\sum_{\mathbf{k}} \gamma_{d} D\left[\hat{d}_{\mathbf{k}}\right]
$$



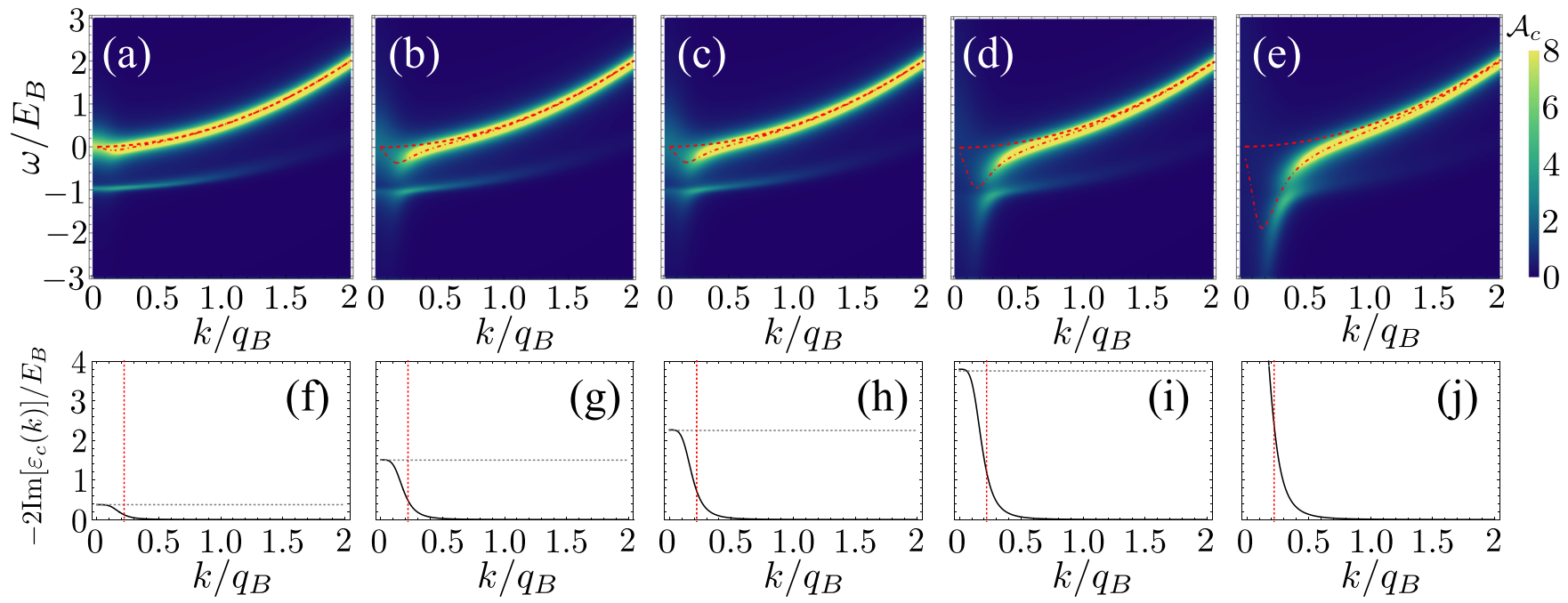

FIG. 7. (Upper row) The spectral function $\mathcal{A}_{c}(\mathbf{k}, \omega)$ of the impurity polariton and (lower row) its decay rate (without interactions with the fermions) for different Rabi frequencies $\Omega_{R}: \Omega_{R} / E_{B}=5.59,11.18,13.69,17.68,25.00$ [from (a) to (e) and from (f) to (j)]. The Fermi energy $\epsilon_{F}=k_{F}^{2} / 2 m_{f}$ is set by $k_{F}=0.22 q_{B}$. The polariton mixes an excitonic state $c$ (or stable internal state in the atomic context) with a photonic mode $d$ (or short-lived internal state in the atomic context). The short-lived state is characterized by the decay rate $\gamma_{d}=333 E_{B}$ and mass $m_{d} / m_{c}=10^{-4}$; the fermion mass is $m_{f}=m_{c}$. The offset is set to zero, $\delta=0$. In (a)-(e), the dashed red line is the dispersion relation $\epsilon_{c}(k)=k^{2} / 2 m_{c}$ of the exciton, the dotted-dashed red line shows the lower polariton branch $\operatorname{Re}\left[\varepsilon_{c}(k)\right]$, which for large $k$ approaches $\epsilon_{c}(k)$. In (f)-(j), the dotted vertical red line is $k_{F}$, and the dotted horizontal gray line is $4 \Omega_{R}^{2} / \gamma_{d}$.

The model just introduced also describes atoms featuring a (meta)stable internal electronic state coupled by a laser to a short-lived excited state, so that $\delta$ is the detuning between the laser frequency and the atomic transition frequency. A situation where the short-lived state has also a much smaller mass, as is naturally the case for excitons and photons, can be implemented with atoms by trapping the stable state in an optical lattice while leaving the excited state untrapped.

The Lindblad master equation resulting from $H_{\text {eff }}$ and $\mathcal{L}_{\text {diss }}$ translates in the Keldysh approach to the inverse retarded GF:

$$
\left[G_{c d}^{R}\right]^{-1}=\left(\begin{array}{cc}
\omega-\epsilon_{c}(\mathbf{k})+i 0 & -\Omega_{R} \\
-\Omega_{R} & \omega-\left[\epsilon_{d}(\mathbf{k})-\delta\right]+i \gamma_{d} / 2+i 0
\end{array}\right) .
$$

Diagonalization of this matrix leads to the dispersion relation and loss rate of the polaritons. One of the complex eigenvalues, which we denote by $\varepsilon_{c}(\mathbf{k})$, converges to $\epsilon_{c}(\mathbf{k})$ for large $|\mathbf{k}|$; the other converges to $\epsilon_{d}(\mathbf{k})+i \gamma_{d} / 2$ for large $|\mathbf{k}|$ and we denote it with $\varepsilon_{d}(\mathbf{k})$. The real part of the eigenvalue $\varepsilon(\mathbf{k})$ describes the position of the resonance, whereas its imaginary part characterizes its width, and hence the loss rate. For simplicity, we assume that the low momenta are sufficient to describe the dynamics so that we may expand $\epsilon_{c, d}(\mathbf{k}) \approx k^{2} / 2 m_{c, d}$, where $m_{c}$ and $m_{d}$ are the effective masses of the uncoupled particles. Now, by changing the ratio $m_{c} / m_{d}$, the detuning $\delta$, and Rabi frequency $\Omega_{R}$, one can adjust the regions in momentum space where the lossy state $d$ interacts with the long-lived state $c$, thereby tuning the loss profile of the polaritons.

In the actual numerical calculations, discussed below, we employ the same set of Dyson's equations introduced in Sec. II where the impurity dispersion and loss rate are the one of the $c$ polariton (the one showing a loss rate which is larger at small momenta), which corresponds to replacing $\epsilon_{c}(\mathbf{k})+i \gamma(\mathbf{k}) / 2$ with $\varepsilon_{c}(\mathbf{k})$ in $G_{0}^{R}$ of Eq. (A3). Here, we are assuming that only one of the two polariton branches is excited and neglecting the mixing between the branches induced by the interaction with the electrons. We are also neglecting the fact that the polariton-electron coupling strength depends on momentum. The inclusion of these features, which in general leads to appreciable quantitative differences, is however beyond the scope of this work.

In Fig. 7, we show the impurity spectral function $\mathcal{A}_{c}(\mathbf{k}, \omega)$ for different Rabi frequencies [see Figs. 7(a)-7(e)] $\Omega_{R} / E_{B}=$ $5.59,11.18,13.69,17.68,25.00$. The photon (or short-lived state in the atomic context) has a large decay rate $\gamma_{d}=333 E_{B}$ and a small mass $m_{d} / m_{c}=10^{-4}$; the detuning is set to $\delta=0$. In this parameter regime, the maximum decay rate is approximately given by $2 \Omega_{R}^{2} / \gamma_{d}$. The parameters are chosen in such a way to have a steep dispersion relation $\epsilon_{d}(k)$ compared to $\epsilon_{c}(k)$, so that the tails of the polariton loss profile drop on a scale smaller than $E_{B}$. This feature is important to have a welldefined DS and thus an almost forbidden region at large loss rates. The impurity and bath fermions are of the same mass. The Fermi wave vector is $k_{F}=0.22 q_{B}$. In Figs. 7(f) $-7(\mathrm{j})$, we show the polariton loss profiles corresponding to Figs. 7(a)7(e). At small Rabi frequency, the polariton dispersion shows minimal deviations from the original exciton dispersion and has also negligible losses, so that the two polaron-polariton peaks are essentially the same as in Fig. 1(a). By increasing the Rabi frequency the impurity-polariton dispersion develops a pronounced minimum due to the photon admixture. The latter at the same time induces polariton losses concentrated around zero momentum. At sufficiently large $\Omega_{R}$ the quantum Zeno effect creates a forbidden region and the dispersion of the polarons is significantly altered. Upon entering the quantum-Zeno regime, both polaron branches show an 
inversion of their curvature, i.e., of the polaron effective mass from positive to negative. In particular, for the attractive polaron the negative curvature becomes very large in the closest vicinity of the forbidden region, which seems to result from a mutual repulsion between the latter and the polaron branch.

Since the quantum-Zeno Fermi-polaron branch has weight only for finite momenta lying outside the forbidden region, the excitation of finite-momentum states is necessary for experimental probing. In transition metal dichalcogenide monolayer semiconductors, optical excitation cannot transfer momenta which are comparable with the Fermi momentum [39], so that a direct probe of the attractive-polaron branch at large enough momenta seems unfeasible. This portion of the attractivepolaron branch is, however, accessible indirectly via the intermediate excitation of the repulsive-polaron branch at zero momentum and subsequent electron-mediated decay into the attractive branch at momenta larger than $k_{F}$. For ultracold atoms, the radio-frequency spectroscopy [36-38,41] usually employed for probing Fermi polarons cannot be used here if one wants to excite finite-momentum states. Instead, twophoton Raman transitions [59] can be exploited for a direct excitation of the attractive-polaron branch at a given finite momentum.

\section{CONCLUSIONS}

In this work, we have studied the 2D Fermi-polaron problem in presence of impurity loss and drive. Our approach, employing diagrammatic methods for nonequilibrium Green's function on the Keldysh time contour [50,51], allows to consistently take into account dissipation and pump and study the strongly dissipative limit. It provides a framework to determine nonequilibrium distributions of fermions, molecules, and impurities. We emphasize they cannot be determined on phenomenological grounds basing on equilibrium quantum field theory and require self-consistent treatment involving the Keldysh component of the Green's functions. We leave the investigation of these intrinsically nonequilibrium features to future work.

In the strongly dissipative regime, we observed the reemergence of unitary Fermi-polaron dynamics, but with an additional length scale set by the size of the region forbidden by the quantum-Zeno effect. The nontrivial interplay between this scale and the Fermi scale induces large qualitative modifications of the impurity-fermion scattering and consequently of the polaron dispersion. We expect this phenomenology to be generic for situations where the losses single out a region of momentum space. In particular, we have shown this to be naturally the case for exciton-polariton impurities and also possible to engineer for atomic gases.

In the same way as the polaron concept has been instrumental in developing a systematic understanding of fundamental phenomena in quantum many-body physics, polarons undergoing a quantum-Zeno projection provide a step towards the understanding of the many-body physics in presence of strong measurements. In particular, our findings identify the emergence of a scale associated with the Zenoforbidden region in an interacting system as a fundamental and generic ingredient able to give rise to novel collective phenomena.

\section{APPENDIX A: NONEQUILIBRIUM GREEN'S FUNCTIONS}

\section{Impurity, fermions, and pairing}

The nonequilibrium Green's functions (GFs) are the building blocks of our theory. For the details of the theory in the path-integral formulation, and some calculations, we refer the reader to Appendix B. The GFs are defined by the relations [50]

$$
\begin{aligned}
& i G_{\alpha}^{R}\left(x, x^{\prime}\right) \equiv \theta\left(t-t^{\prime}\right)\left\langle\left[\hat{\alpha}(x), \hat{\alpha}^{\dagger}\left(x^{\prime}\right)\right]_{\mp}\right\rangle, \\
& i G_{\alpha}^{K}\left(x, x^{\prime}\right) \equiv\left\langle\left[\hat{\alpha}(x), \hat{\alpha}^{\dagger}\left(x^{\prime}\right)\right]_{ \pm}\right\rangle,
\end{aligned}
$$

where the subscript $+(-)$ indicates the anticommutator (commutator), and $\hat{\alpha}^{\dagger}$ creates a particle in one of the degrees of freedom, that is, for a fermion $\hat{\alpha}^{\dagger}=\hat{f}^{\dagger}$ or for the impurity $\hat{\alpha}^{\dagger}=\hat{c}^{\dagger}$; hereafter, we denote the space-time variable by $x=(\mathbf{r}, t)$. The correlator in (A1a) is the retarded GF, which is nonzero only for $t>t^{\prime}$. Related to $G^{R}$ is the advanced GF denoted by $G_{\alpha}^{A}\left(x, x^{\prime}\right)=\left[G_{\alpha}^{R}\left(x^{\prime}, x\right)\right]^{*}$. The correlator in (A1b) is the so-called Keldysh GF or statistical propagator. While the retarded GF $G^{R}$ contains information only about the excitation spectrum of the system [being the expectation value of a (anti)commutator for bosons (fermions) it is indeed normalized to 1], the Keldysh GF also depends on the occupation of excitation modes. The Keldysh GF is in general independent of $G^{R / A}$ and has to satisfy its own evolution equation. Only in thermal equilibrium the fluctuation-dissipation relation (FDR) allows to express the value of $G^{K}$ in terms of $G^{R}$ and $G^{A}$. It is only in this case that the latter are sufficient for the description of the problem. Away from thermal equilibrium, the introduction of the Keldysh GF is necessary and in particular in driven-dissipative systems even in stationary states [51].

In our system, only the impurities are subject to loss and gain, while the fermions are stable particles. Thus, since the number of impurities is very small compared to the density of fermions, we can assume that the fermions remain in a thermal equilibrium state. The Keldysh GF can then be related by FDR to the remaining two GFs as follows:

$$
G_{f}^{K}(p)=F_{f}^{\mathrm{eq}}(\omega)\left[G_{f}^{R}(p)-G_{f}^{A}(p)\right],
$$

with the thermal distribution $F_{f}^{\mathrm{eq}}(\omega)=1-2 n_{f}(\omega)$ where $n_{f}(\omega)=\theta\left(\epsilon_{F}-\omega\right)$ is the Fermi-Dirac distribution at $T=0$, $\theta$ the unit step function, and $\epsilon_{F}$ the Fermi energy. Using the time- and space-translation invariance in the steady state, all GFs are expressed by their Fourier transforms in the relative coordinate, for example, $G_{f}^{R}(x, 0) \equiv G_{f}^{R}(\mathbf{r}, t) \equiv G_{f}^{R}(x)=$ $\sum_{p} e^{i p \cdot x} G_{f}^{R}(p)$ and, hereafter, following Ref. [50], we denote the momentum-energy vector $p=(\mathbf{k}, \omega)$, and the scalar product $p \cdot x=\mathbf{k} \cdot \mathbf{r}-\omega t$; the sum here is $\sum_{p}=\sum_{\mathbf{k}} \int d \omega / 2 \pi$. Finally, the retarded (advanced) GF is given by $G_{f}^{R / A}=$ $\left[\omega-\varepsilon_{f}(\mathbf{k}) \pm i 0\right]^{-1}$, where the infinitesimal imaginary number, denoted with $\pm i 0$, ensures the proper support in real time.

Neglecting the impurity-fermion interaction, it is straightforward to evaluate the impurity GFs. These GFs, which are the building blocks for our diagrammatic approach, are referred to as "bare" (denoted by an additional subscript 0 ). Since we assume that the fermions are not renormalized by 
the presence of a small number of impurities, we drop the subscript "0" from fermionic GFs. The impurity GFs read as

$$
\begin{aligned}
G_{0, c}^{R / A}(\mathbf{k}, \omega) & =\frac{1}{\omega-\varepsilon_{c}(\mathbf{k}) \pm i \frac{\gamma(\mathbf{k})}{2}}, \\
G_{0, c}^{K}(\mathbf{k}, \omega) & =F_{0, c}(\mathbf{k})\left[G_{0, c}^{R}(p)-G_{0, c}^{A}(p)\right],
\end{aligned}
$$

with $F_{0, c}(\mathbf{k})=1+2 n_{0, c}(\mathbf{k})$ and $n_{0, c}(\mathbf{k})=\Omega(\mathbf{k}) / \gamma(\mathbf{k})$. The distribution function $F_{0, c}$ has the same structure as $F_{f}^{\text {eq }}$ (the opposite relative sign is due to the bosonic statistics for the impurities). However, in place of the thermal occupation the ratio of pump to loss rate appears in $F_{0, c}$. This ratio gives the maximal number of impurities $n_{0, c}(\mathbf{k})$ allowed to occupy a given momentum state $\mathbf{k}$.

In the Fermi-polaron problem, bound-state formation between the impurity and a bath-fermion plays a prominent role [60]. Thus, featuring a pole already in the two-body problem, the particle-particle scattering vertex dominates the interactions in the Fermi-polaron problem. To capture this pairing channel we introduce an auxiliary molecular field, which provides access to molecular degrees of freedom having the bound state as a long-lived excitation. As already known from thermal equilibrium, this approach to the interactions between impurity and bath is fully equivalent to the $T$-matrix approach. As we show in Appendix B, the molecular field can be properly introduced within the Keldysh functional-integral formalism by performing a Hubbard-Stratonovich transformation. This leads to the following interaction part of the functional-integral action:

$$
S_{\Delta, f, c}=-\int_{\mathcal{C}} d t \int d \mathbf{r}(\bar{c} \bar{f} \Delta+\bar{\Delta} f c),
$$

where $\mathcal{C}$ is the Keldysh time contour [50]. The molecular field $\Delta$ introduced via the Hubbard-Stratonovich transformation has actually no dynamics, the latter being generated only by the interaction with impurities and fermions. Formally, this translates in the bare molecular propagator being simply given by $G_{0, \Delta}^{R / A}=(1 / U \pm i 0)^{-1}$. The noninteracting Keldysh component of the molecular action is vanishingly small and is overwritten by the interaction corrections as expressed by the self-energies, which we introduce next.

\section{Dyson equation and diagrammatic expansion}

The interactions in the system lead to the modification (so-called dressing) of the bare GFs. We compute these corrections from Dyson's equation (for details see Appendix B 3). Within the Keldysh formalism, one has to solve two coupled Dyson's equations for the retarded and the Keldysh GFs, respectively, since, as explained above, the two are in general independent when the out-of-equilibrium case is considered. In what follows, we are interested in the spectral signatures of the polarons, which are contained in the retarded, dressed GF of the impurity

$$
G_{c}^{R}(\mathbf{k}, \omega)=\frac{1}{\omega-\varepsilon_{c}(\mathbf{k})+i \frac{\gamma(\mathbf{k})}{2}-\Sigma_{c}^{R}(p)+i 0} .
$$

Here, the retarded self-energy $\Sigma_{c}^{R}$ results from the interactions, the relevant process being the one where the impurity scatters with a fermion, temporarily forms a molecule, which
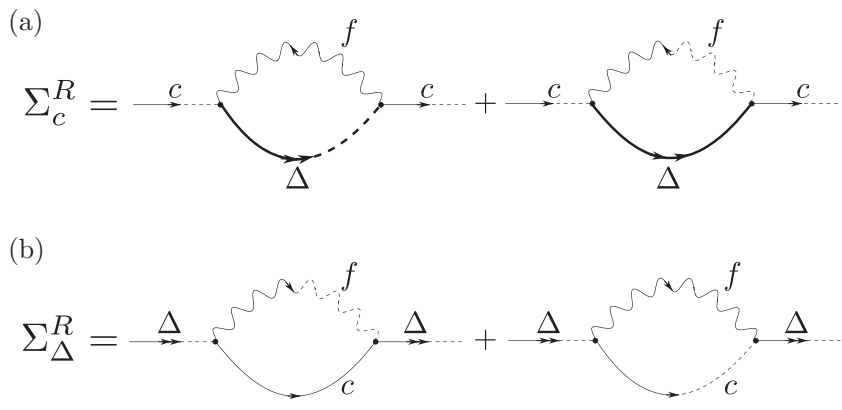

FIG. 8. The diagrams showing the scattering processes that give rise to the impurity (a) and the molecule (b) self-energies (after truncation of external lines). The Keldysh structure, i.e., the types of Green's functions, is explicitly presented (cf. Table I). In (a), during propagation of the impurity, the particle scatters with a fermion $f$ to form a molecule $\Delta$, and then the molecule decays back into the fermion and the impurity. This process yields $\Sigma_{c}^{R}$. In (b), a propagating molecule decays into a fermion and the impurity, which subsequently collide to form the molecule. This process gives rise to $\Sigma_{\Delta}^{R}$. To highlight the compound molecular structure, we denote its propagator with the line with two arrows.

then decays back into an impurity and a fermion. This process is displayed in Fig. 8(a) in terms of Feynman diagrams, where, as summarized in Table I, each line corresponds to a GF and the intersection points to interaction vertices. In particular, dressed and bare GFs are depicted as thick and thin lines, respectively. The choice of the diagrams in Fig. 8 is a direct generalization of a non-self-consistent $T$-matrix approach to a driven-dissipative setting. In particular, if we assume thermal equilibrium, i.e., set the impurity losses to zero, it reproduces the result of the non-self-consistent $T$-matrix approach in the impurity limit.

Every diagram corresponds to a mathematical expression for the self-energy that can be obtained as follows. To each line an energy-momentum vector is assigned. For internal lines, the latter is taken as the argument of the GF, while for the external lines it sets the argument of the self-energy we are computing. At each vertex energy and momentum

TABLE I. Diagrammatic representation of the Green's functions for the impurity (left column), fermions (middle column), and molecules (right column). To each line energy and momentum variables are attached, which are taken as the argument of the corresponding Green's function. The double arrows on the molecular lines indicate their composite nature. Keldysh GFs are drawn with solid lines, whereas solid-dashed (dashed-solid) lines represent retarded (advanced) Green's functions.

\begin{tabular}{ccc}
\hline \hline Impurity & Fermion & Molecule \\
\hline$G_{c}^{R}-G_{f}^{R}$ & $G_{\Delta}^{R}-G_{\Delta}^{A}$ \\
$\longrightarrow G_{c}^{A}$ & $G_{f}^{A}$ & $-G_{\Delta}^{K}$ \\
\hline
\end{tabular}


conservation is imposed. Finally, a sum is performed over all energy-momentum vectors of the internal lines.

With this procedure, we obtain from Fig. 8(a) the following self-energy:

$\Sigma_{c}^{R}(p)=-\frac{i}{2 V} \sum_{p^{\prime}}\left[G_{f}^{K}\left(p^{\prime}\right) G_{\Delta}^{R}\left(p+p^{\prime}\right)+G_{\Delta}^{K}\left(p^{\prime}\right) G_{f}^{A}\left(p^{\prime}-p\right)\right]$,

where $V$ is the area of the two-dimensional system. The advanced and retarded GF of each particle is always multiplied by a Keldysh GF of the other particle, which carries information about occupations of the states.

In order to compute the impurity self-energy from Eq. (A6), we need the molecular GF. The retarded component is given by

$$
G_{\Delta}^{R}(p)=\frac{1}{-v^{2}-\Sigma_{\Delta}^{R}(p)+i 0},
$$

with $v^{2}=-1 / U$ and $\Sigma_{\Delta}^{R}(p)$ the molecular self-energy. The molecular Keldysh GF can be parametrized again as $G_{\Delta}^{K}=$ $F_{\Delta}\left(G_{\Delta}^{R}-G_{\Delta}^{A}\right)$. If the number of impurities is very small, the number of molecules is also very small, resulting in $F_{\Delta} \approx 1$. In the impurity limit and assuming the Fermi bath to be at $T=0$, we thus have

$$
\Sigma_{c}^{R}(\mathbf{k}, \omega)=\frac{1}{V} \sum_{\mathbf{k}^{\prime} \in \mathrm{FS}} \frac{1}{-v^{2}-\Sigma_{\Delta}^{R}\left(\mathbf{k}+\mathbf{k}^{\prime}, \omega+\varepsilon_{f}\left(\mathbf{k}^{\prime}\right)\right)+i 0},
$$

where the summation is restricted to momenta from inside the Fermi sea (FS) for which $\left|\mathbf{k}^{\prime}\right| \leqslant \epsilon_{F}$. This constraint on the fermion momenta is a direct consequence of the presence of $G_{f}^{K}$ in Eq. (A6). Physically, during the propagation of the impurity a transient molecular state can be formed if the impurity collides with a fermion, and this can be taken only from the Fermi sea.

The molecular self-energy $\Sigma_{\Delta}^{R}(p)$ is needed to evaluate $\Sigma_{c}^{R}(p)$ and in turn $G_{c}^{R}(p)$. The dominant process that leads to the modification of the propagation of a free molecule is depicted in Fig. 8(b). A propagating molecule decays virtually into an impurity and a fermion, which subsequently collide to form the molecule again. The expression for the self-energy resulting from the Feynman diagram reads as

$\Sigma_{\Delta}^{R}(p)=\frac{i}{2 V} \sum_{p^{\prime}}\left[G_{0, c}^{K}\left(p^{\prime}\right) G_{f}^{R}\left(p-p^{\prime}\right)+G_{f}^{K}\left(p^{\prime}\right) G_{0, c}^{R}\left(p-p^{\prime}\right)\right]$,

where we use the bare impurity GFs. Since all functions entering the equation for $\Sigma_{\Delta}^{R}$ are known analytically, the selfenergy can be computed explicitly in the impurity limit:

$$
\Sigma_{\Delta}^{R}(\mathbf{k}, \omega)=\frac{1}{V} \sum_{\mathbf{k}^{\prime} \notin \mathrm{FS}} \frac{1}{\omega-\varepsilon_{c}\left(\mathbf{k}-\mathbf{k}^{\prime}\right)-\varepsilon_{f}\left(\mathbf{k}^{\prime}\right)+i \frac{\gamma\left(\mathbf{k}-\mathbf{k}^{\prime}\right)}{2}+i 0} .
$$

Notice that the sum over momenta extends outside the Fermi sea. This is a consequence of the fact that the propagating molecule can decay into an impurity-fermion pair, but due to Pauli blocking, the fermion cannot occupy any state from the Fermi sea. Due to the composite nature of the molecule, its GF is affected by impurity loss. In turn, the impurity self-energy in Eq. (A8) is affected by loss through $\Sigma_{\Delta}^{R}$. Since $G_{0, c}^{K}\left(p^{\prime}\right)$ does not depend on $\Omega(\mathbf{k})$ in the impurity limit, there is no pump dependence left in any of the retarded GFs, which are discussed in the main text.

Finally, let us make more explicit the connection between the above results and the known case of the lossless Fermi polaron. For this purpose, let us consider the quasiparticle excitations of the system, whose energy and damping is given by the complex poles of the retarded impurity GF. By setting $\gamma(\mathbf{k})$ to zero we obtain the following equation determining the quasiparticle dispersion: $\omega(\mathbf{k})=\varepsilon_{c}(\mathbf{k})-\Sigma_{c}^{R}(\mathbf{k}, \omega(\mathbf{k}))$. Exactly the same equation $[30,35,44,47]$ is derived when the variational approach is employed with the Chevy polaron ansatz $|\Psi(\mathbf{k})\rangle=\chi \hat{c}_{\mathbf{k}}^{\dagger}|\mathrm{FS}\rangle+\sum_{\mathbf{k}^{\prime}, \mathbf{q}} \chi_{\mathbf{k}^{\prime} \mathbf{q}} \hat{c}_{\mathbf{k}-\mathbf{k}^{\prime}+\mathbf{q}}^{\dagger} \hat{f}_{\mathbf{k}^{\prime}}^{\dagger} \hat{f}_{\mathbf{q}}|\mathrm{FS}\rangle$, where $\mathbf{k}^{\prime} \notin$ FS and $\mathbf{q} \in$ FS. This trial wave function, with variational parameters $\chi$ and $\chi_{\mathbf{k}^{\prime} \mathbf{q}}$, includes single particle-hole excitations and is able to capture the influence of the molecular state on the impurity propagation.

\section{APPENDIX B: KELDYSH PATH-INTEGRAL FORMULATION OF THE THEORY}

In our work, we follow the approach presented in Refs. [50,51]. The quantum average of any operator can be calculated using the path-integral formulation. Here, the quantum averages over a state, given by a density matrix, are calculated by summing over all possible realizations of fields weighted by a complex exponent that can be interpreted as an action. Below, we present the structure of the theory and derive the formulas presented in the main text.

We first consider the impurities. Although the statistics of the impurities is irrelevant, we choose them to be bosons since this simplifies the functional-integral formulation. We then treat the fermionic bath using Grassmann fields. Finally, we include the interactions between impurities and fermions, which can be reformulated by introducing the molecular fields and their interaction vertex with fermions and impurities.

\section{Partition function}

Our starting point is the partition function of the system, written as an integral over field configurations:

$$
Z=\int \mathcal{D}[c, f] e^{i S\left[\bar{c}_{+}, c_{+}, \bar{c}_{-}, c_{-}, \bar{f}_{+}, f_{+}, \bar{f}_{-}, f_{-}\right]},
$$

where for simplicity in the integration measure $\mathcal{D}[c, f] \equiv$ $\mathcal{D}\left[\bar{c}_{+}, c_{+}, \bar{c}_{-}, c_{-}, \bar{f}_{+}, f_{+}, \bar{f}_{-}, f_{-}\right]$we suppressed dependence on position (or momentum) and time of the fields. The action $S=S_{c}+S_{f}+S_{\text {int }}$ can be decomposed into three parts that describe the bosonic impurities, fermions, and the interaction, respectively. Below, we describe them separately.

The bosonic impurities are governed by

$$
\begin{aligned}
S_{c}= & \int d t \sum_{\mathbf{k}}\left\{\bar{c}_{+}\left[i \partial_{t}-\varepsilon_{c}(\mathbf{k})\right] c_{+}-\bar{c}_{-}\left[i \partial_{t}-\varepsilon_{c}(\mathbf{k})\right] c_{-}\right. \\
& -i[\gamma(\mathbf{k})+\Omega(\mathbf{k})]\left[c_{+} \bar{c}_{-}-\frac{1}{2}\left(\bar{c}_{+} c_{+}+\bar{c}_{-} c_{-}\right)\right] \\
& \left.-i \Omega(\mathbf{k})\left[\bar{c}_{+} c_{-}-\frac{1}{2}\left(\bar{c}_{+} c_{+}+\bar{c}_{-} c_{-}\right)\right]\right\}
\end{aligned}
$$

where the complex-valued field $c_{ \pm} \equiv c_{ \pm}(\mathbf{k}, t)$ resides on the forward (backward) branch of the Keldysh contour $\mathcal{C}$, which 
goes from $t=-\infty$ to $t=\infty$ (forward branch) and then from $t=+\infty$ to $t=-\infty$ (backward branch). Next, we perform the Keldysh rotation according to

$$
c_{c l / q}=\frac{c_{+} \pm c_{-}}{\sqrt{2}} \quad \text { and } \quad \bar{c}_{c l / q}=\frac{\bar{c}_{+} \pm \bar{c}_{-}}{\sqrt{2}},
$$

where the indices $c l$ and $q$ stand for "classical" and "quantum," respectively. Since we are interested in the stationary states, we perform the Fourier transform and express the fields in terms of the frequency variable. In the new basis, the action takes the form

$$
S_{c}=\sum_{p} \bar{c}_{\alpha}(p) G_{0, c, \alpha \alpha^{\prime}}^{-1}(p) c_{\alpha^{\prime}}(p),
$$

where the sum over repeated indices is implied here and in the following. The object $G_{0, c, \alpha \alpha^{\prime}}^{-1}(p)$ is the bare inverse of the impurity GF, which reads as

$$
G_{0, c}^{-1}=\left(\begin{array}{cc}
0 & {\left[G_{0, c}^{A}\right]^{-1}} \\
{\left[G_{0, c}^{R}\right]^{-1}} & {\left[G_{0, c}^{-1}\right]^{K}}
\end{array}\right) .
$$

We note that the $q$ - $q$ term is the Keldysh component of the inverse of the GF, and its inverse is not equal to the Keldysh component of the GF (see below). The components of the matrix are given by

$$
\begin{aligned}
& {\left[G_{0, c}^{R / A}\right]^{-1}=\omega-\varepsilon_{c}(\mathbf{k}) \pm i \frac{\gamma(\mathbf{k})}{2},} \\
& {\left[0 G_{0, c}^{-1}\right]^{K}=i[\gamma(\mathbf{k})+\Omega(\mathbf{k})] .}
\end{aligned}
$$

The inversion of Eq. (B7) results in Eq. (A3). In this way, we have

$$
G_{0, c}=\left(\begin{array}{cc}
G_{0, c}^{K} & G_{0, c}^{R} \\
G_{0, c}^{A} & 0
\end{array}\right),
$$

and the entries are given by

$$
\begin{aligned}
G_{0, c, \alpha \alpha^{\prime}}(p) & =\left\langle c_{\alpha}(p) \bar{c}_{\alpha^{\prime}}(p)\right\rangle \\
& =\int \mathcal{D}[\bar{c}, c] c_{\alpha}(p) \bar{c}_{\alpha^{\prime}}(p) e^{i S_{c}[c, \bar{c}]} .
\end{aligned}
$$

We emphasize that in the Keldysh formulation of the theory the $c l$ - $c l$ component of $G_{0, c}^{-1}$ is zero. This property applies also to the full dressed GF when the interactions are included and the average is taken over the total action $S$. This is referred to as the "causal structure" and originates from the detailed derivation of the path-integral formalism [50]. The causal structure of the theory is reflected in the vanishing of $q-q$ component of the GF.

Now, we proceed to the fermionic action given by

$$
S_{c}=\int_{\mathcal{C}} d t \sum_{\mathbf{k}} \bar{f}(\mathbf{k}, t)\left[i \partial_{t}-\varepsilon_{f}(\mathbf{k})\right] f(\mathbf{k}, t) .
$$

Here, the fields $f$ and $\bar{f}$ are independent Grassmann noncommuting numbers. Next, we write explicitly the fields on forward and backward branches of the Keldysh contour, and perform the Keldysh rotation according to

$$
f_{ \pm}=\frac{f_{1} \pm f_{2}}{\sqrt{2}} \quad \text { and } \quad \bar{f}_{ \pm}=\frac{f_{2} \mp f_{1}}{\sqrt{2}} .
$$

As a result, after taking the Fourier transform, we obtain the action

$$
S_{f}=\sum_{p} \bar{f}_{a}(p) G_{f, a a^{\prime}}^{-1}(p) f_{a^{\prime}}(p),
$$

where the Keldysh indices $a$ and $a^{\prime}$ can be either 1 or 2 . The inverse of the fermionic GF is given by

$$
G_{f}^{-1}=\left(\begin{array}{cc}
{\left[G_{f}^{R}\right]^{-1}} & {\left[G_{f}^{-1}\right]^{K}} \\
0 & {\left[G_{f}^{A}\right]^{-1}}
\end{array}\right),
$$

which, after inversion, leads to the following GF:

$$
G_{f}=\left(\begin{array}{cc}
G_{f}^{R} & G_{f}^{K} \\
0 & G_{f}^{A}
\end{array}\right),
$$

and, assuming fermions of infinite lifetime, the entries are given by $G_{f}^{R / A}(p)=\left[\omega-\varepsilon_{f}(\mathbf{k}) \pm i 0\right]^{-1}$ and $G_{f}^{K}$ is in Eq. (A2). The causal structure of the theory leads to $\left[G_{f}\right]_{21} \equiv 0$.

\section{Interaction and molecules}

The action corresponding to the interaction in Eq. (2) is given by

$$
S_{\mathrm{int}}=-U \int_{\mathcal{C}} d t \int d \mathbf{r} \bar{c}(x) c(x) \bar{f}(x) f(x) .
$$

We proceed with the Hubbard-Strantonovich transformation that introduces the molecular field which mediates the contact interaction in Eq. (B17).

To this end, we introduce an auxiliary fermionic field $\Delta(x)$ that decouples the interaction potential:

$$
e^{i S_{\mathrm{int}}}=\int \mathcal{D}[\bar{\Delta}, \Delta] e^{i S_{\Delta}+i S_{\Delta, f, c}},
$$

with

$$
\begin{aligned}
S_{\Delta} & =\int_{\mathcal{C}} d t \int d \mathbf{r} \bar{\Delta}(x) U^{-1} \Delta(x), \\
S_{\Delta, f, c} & =-\int_{\mathcal{C}} d t \int d \mathbf{r}(\bar{c} \bar{f} \Delta+\bar{\Delta} f c) .
\end{aligned}
$$

Now, the action is linear in the fermionic fields, and thus the fermionic degrees of freedom can be integrated out. Consequently, we write

$$
\int \mathcal{D}[\bar{f}, f] e^{i S_{f}+i S_{\Delta, f, c}}=e^{i S_{\Delta c}},
$$

where the effective resulting action is given by

$$
\begin{aligned}
S_{\Delta c}= & -\frac{1}{2} \int d x^{\prime} \int d x^{\prime} \bar{c}_{\beta}\left(x^{\prime}\right) \Delta_{u}(x) \gamma_{a, u}^{\alpha} \\
& \times G_{f, a b}\left(x, x^{\prime}\right) \gamma_{b, v}^{\beta} \Delta_{v}\left(x^{\prime}\right) c_{\alpha}(x) .
\end{aligned}
$$

The sum over indices $a, b, u, v$ runs over 1 and 2 , whereas the sum over $\alpha, \beta$ runs over $c l$ and $q$. The matrix $\gamma^{c l}$ is the identity matrix in the Keldysh indices, i.e., $\gamma_{a, b}^{c l}=\delta_{a, b}$, and $\gamma^{q}$ has unit entries on the off-diagonal, i.e., $\gamma_{a b}^{q}=1-\delta_{a, b}$. Finally, the molecular action $S_{\Delta}$ leads to the inverse bare molecular GF:

$$
G_{0, \Delta}^{-1}=\left(\begin{array}{cc}
{\left[G_{0, \Delta}^{R}\right]^{-1}} & {\left[G_{0, \Delta}^{-1}\right]^{K}} \\
0 & {\left[G_{0, \Delta}^{A}\right]^{-1}}
\end{array}\right),
$$




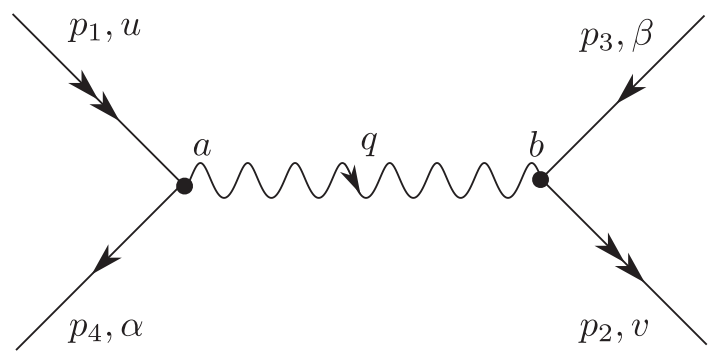

FIG. 9. The vertex generated by the action $S_{\Delta c}$ [see Eq. (B24)], describing interaction between a molecule (denoted with a solid line with a double arrow) and impurities (denoted with a solid line with a single arrow). Each line carries the momentum variable and the Keldysh index. The interaction mediated by fermions is denoted by the wavy line, which is labeled by a momentum variable $q$ and two Keldysh indices [together they determine $G_{f, a b}(q)$ ]. To the diagram we assign the expression that is shown in Eq. (B25).

where $\left[G_{0, \Delta}^{R / A}\right]^{-1}=1 / U \pm i 0$. The action in the Keldysh indices and in momentum space can be written as $S_{\Delta}=$ $\sum_{p} \bar{\Delta}_{a}(p) G_{0, \Delta, a b}^{-1}(p) \Delta_{b}(p)$; here $a, b$ takes values 1 or 2 . The off-diagonal Keldysh component is vanishingly small for the bare propagator, but it is renormalized when the interactions are taken into account.

Going back to the momentum representation the action reads as

$$
\begin{aligned}
S_{\Delta c}= & -\frac{1}{2 V} \sum_{p_{1}, p_{2}, p_{3}, p_{4}}^{\prime} G_{f, a b}\left(p_{1}-p_{4}\right) \gamma_{u, a}^{\alpha} \gamma_{b, v}^{\beta} \\
& \times \bar{\Delta}_{u}\left(p_{1}\right) \Delta_{v}\left(p_{2}\right) \bar{c}_{\beta}\left(p_{3}\right) c_{\alpha}\left(p_{4}\right),
\end{aligned}
$$

where the prime on the sum sign indicates the constraint $p_{1}+p_{2}=p_{3}+p_{4}$; the sum over repeated discrete indices $a, b, u, v, \alpha, \beta$ is implicit.

In Fig. 9, we display the vertex generated by the action $S_{\Delta c}$ defined by Eq. (B24). Each line carries a momentum and a Keldysh index. A solid line with an arrow denotes the impurity field, while double arrows represent the molecular fields. When forming diagrams, two lines are connected to form a GF that, depending on the carried Keldysh indices, is taken as the retarded or advanced or Keldysh GF, as shown in Table I. In the figure, the wavy line stands for the fermion GF. It carries an index at both ends, denoted by $a$ and $b$, and a momentum $q$. The diagram is thus translated into the expression

$$
-\frac{1}{2} \frac{1}{V} \gamma_{u, a}^{\alpha} G_{f, a b}(q) \gamma_{b, v}^{\beta},
$$

where $q=p_{1}-p_{4}=p_{2}-p_{3}$.

From the vertex shown in Fig. 9 we can evaluate the selfenergies. To calculate the molecular self-energy, we connect the external impurity lines, i.e., we set $p_{3}=p_{4}$, and introduce $G_{0, c, \alpha \beta}\left(p_{3}\right)$ for that line. In this way, we arrive at the self-energy shown in Fig. 8(b). When the sum over Keldysh indices is evaluated, we obtain a sum of diagrams in which retarded or advanced and Keldysh GFs appear [see Fig. 8(b)]. Analogously, by joining the molecular line, i.e., by setting $p_{1}=p_{2}$ and introducing $G_{\Delta, u v}\left(p_{1}\right)$, we obtain the diagram from Fig. 8(a). Taking the sum over the Keldysh indices we arrive at the sum of diagrams [see Fig. 8(a)]. However, in the resummation scheme employed in this work, in the calculation of the impurity self-energy the dressed molecular GF is used instead of the bare one.

\section{Dyson equation}

The action of the system, after tracing out the fermions and introducing the molecular channel consists of three parts

$$
S=S_{c}+S_{\Delta}+S_{\Delta c} .
$$

The first two terms on the right-hand side generate the impurity and molecular bare Green functions, respectively. The third term is responsible for the conversion of a fermion and an impurity in an impurity-fermion molecule.

The evaluation of the dressed GFs, which are given by average over the field weighted by $e^{i S}$, can be understood as averaging over $e^{i S_{c}+i S_{\Delta}}$ and an expansion of $e^{i S_{\Delta c}}$ in powers of the interaction. The usual diagrammatic formulation of the theory is then recovered when we assign lines to the GFs as shown in Table I.

As a result of the summation of the diagrams, we obtain Dyson equations for the GFs:

$$
\begin{aligned}
G_{c} & =G_{0, c}+G_{0, c} \cdot \Sigma_{c} \cdot G_{c}, \\
G_{\Delta} & =G_{0, \Delta}+G_{0, \Delta} \cdot \Sigma_{\Delta} \cdot G_{\Delta},
\end{aligned}
$$

where by the center dot we denote the multiplication of the Keldysh matrices. Here, the self-energies $\Sigma_{c}$ and $\Sigma_{\Delta}$ are matrices generated by irreducible diagrams for the impurity and molecular GFs, respectively. They have a causal structure similar to the inverse GFs [50]:

$$
\Sigma_{\Delta}=\left(\begin{array}{cc}
\Sigma_{\Delta}^{R} & \Sigma_{\Delta}^{K} \\
0 & \Sigma_{\Delta}^{A}
\end{array}\right)
$$

for the molecule, and

$$
\Sigma_{c}=\left(\begin{array}{cc}
0 & \Sigma_{c}^{A} \\
\Sigma_{c}^{R} & \Sigma_{c}^{K}
\end{array}\right)
$$

for the impurity.

The self-energy of the molecule is generated by the diagram shown in Fig. 8(b). Employing the vertex from the previous Appendix, we arrive at

$$
\begin{aligned}
\Sigma_{\Delta, a b}(p)= & \frac{i}{2 V} \sum_{p^{\prime}} \gamma_{a a^{\prime}}^{\alpha} \gamma_{b b^{\prime}}^{\beta} \\
& \times G_{f, a^{\prime} b^{\prime}}\left(p-p^{\prime}\right) G_{0, c, \alpha \beta}\left(p^{\prime}\right) .
\end{aligned}
$$

The retarded self-energy is thus given by $\Sigma_{\Delta}^{R}=\left[\Sigma_{\Delta}\right]_{11}$, which leads to Eq. (A9).

Analogously, the diagram from Fig. 8 generates the selfenergy for the impurity. Here, we take the dressed GFs for the molecule with self-energy given by (B30). As a result, we obtain

$$
\Sigma_{x}^{\alpha \beta}(p)=\frac{-i}{2 V} \sum_{p^{\prime}} \operatorname{Tr}\left[\gamma^{\beta} \cdot G_{f}\left(p^{\prime}\right) \cdot \gamma^{\alpha} \cdot G_{\Delta}\left(p+p^{\prime}\right)\right],
$$

where the trace acts in the Keldysh indices. Finally, using Eq. (B29) we find $\Sigma_{x}^{R}=\left[\Sigma_{x}\right]_{q, c l}$, from which follows Eq. (A6). 
[1] H.-P. Breuer and F. Petruccione, The Theory of Open Quantum Systems (Oxford University Press, Oxford, 2002).

[2] H. M. Wiseman and G. J. Milburn, Quantum Measurement and Control (Cambridge University Press, Cambridge, 2009).

[3] B. Misra and E. C. G. Sudarshan, The Zeno's paradox in quantum theory, J. Math. Phys. 18, 756 (1977).

[4] W. M. Itano, D. J. Heinzen, J. J. Bollinger, and D. J. Wineland, Quantum Zeno effect, Phys. Rev. A 41, 2295 (1990).

[5] C. Presilla, R. Onofrio, and U. Tambini, Measurement quantum mechanics and experiments on quantum Zeno effect, Ann. Phys. 248, 95 (1996).

[6] P. Facchi and S. Pascazio, Quantum Zeno Subspaces, Phys. Rev. Lett. 89, 080401 (2002).

[7] T. J. Elliott, W. Kozlowski, S. F. Caballero-Benitez, and I. B. Mekhov, Multipartite Entangled Spatial Modes of Ultracold Atoms Generated and Controlled by Quantum Measurement, Phys. Rev. Lett. 114, 113604 (2015).

[8] G. Mazzucchi, W. Kozlowski, S. F. Caballero-Benitez, T. J. Elliott, and I. B. Mekhov, Quantum measurement-induced dynamics of many-body ultracold bosonic and fermionic systems in optical lattices, Phys. Rev. A 93, 023632 (2016).

[9] S. Dhar and S. Dasgupta, Measurement-induced phase transition in a quantum spin system, Phys. Rev. A 93, 050103(R) (2016).

[10] X. Cao, A. Tilloy, and A. De Luca, Entanglement in a fermion chain under continuous monitoring, Sci. Post. Phys. 7, 24 (2019).

[11] Y. Li, X. Chen, and M. P. A. Fisher, Quantum Zeno effect and the many-body entanglement transition, Phys. Rev. B 98, 205136 (2018).

[12] A. Chan, R. M. Nandkishore, M. Pretko, and G. Smith, Unitaryprojective entanglement dynamics, Phys. Rev. B 99, 224307 (2019).

[13] B. Skinner, J. Ruhman, and A. Nahum, Measurement-Induced Phase Transitions in the Dynamics of Entanglement, Phys. Rev. X 9, 031009 (2019).

[14] N. Katz, M. Ansmann, R. C. Bialczak, E. Lucero, R. McDermott, M. Neeley, M. Steffen, E. M. Weig, A. N. Cleland, J. M. Martinis, and A. N. Korotkov, Coherent state evolution in a superconducting qubit from partial-collapse measurement, Science 312, 1498 (2006).

[15] R. Vijay, C. Macklin, D. H. Slichter, S. J. Weber, K. W. Murch, R. Naik, A. N. Korotkov, and I. Siddiqi, Stabilizing Rabi oscillations in a superconducting qubit using quantum feedback, Nature (London) 490, 77 (2012).

[16] P. Campagne-Ibarcq, P. Six, L. Bretheau, A. Sarlette, M. Mirrahimi, P. Rouchon, and B. Huard, Observing Quantum State Diffusion by Heterodyne Detection of Fluorescence, Phys. Rev. X 6, 011002 (2016).

[17] N. Syassen, D. M. Bauer, M. Lettner, T. Volz, D. Dietze, J. J. Garcia-Ripoll, J. I. Cirac, G. Rempe, and S. Dürr, Strong dissipation inhibits losses and induces correlations in cold molecular gases, Science 320, 1329 (2008).

[18] S. Dürr, J. J. García-Ripoll, N. Syassen, D. M. Bauer, M. Lettner, J. I. Cirac, and G. Rempe, Lieb-Liniger model of a dissipation-induced Tonks-Girardeau gas, Phys. Rev. A 79, 023614 (2009).

[19] J. J. García-Ripoll, S. Dürr, N. Syassen, D. M. Bauer, M. Lettner, G. Rempe, and J. I. Cirac, Dissipation-induced hard- core boson gas in an optical lattice, New J. Phys. 11, 013053 (2009).

[20] G. Barontini, R. Labouvie, F. Stubenrauch, A. Vogler, V. Guarrera, and H. Ott, Controlling the Dynamics of an Open Many-Body Quantum System with Localized Dissipation, Phys. Rev. Lett. 110, 035302 (2013).

[21] R. Labouvie, B. Santra, S. Heun, and H. Ott, Bistability in a Driven-Dissipative Superfluid, Phys. Rev. Lett. 116, 235302 (2016).

[22] B. Yan, S. A. Moses, B. Gadway, J. P. Covey, K. R. A. Hazzard, A. M. Rey, D. S. Jin, and J. Ye, Observation of dipolar spinexchange interactions with lattice-confined polar molecules, Nature (London) 501, 521 (2013).

[23] B. Zhu, B. Gadway, M. Foss-Feig, J. Schachenmayer, M. L. Wall, K. R. A. Hazzard, B. Yan, S. A. Moses, J. P. Covey, D. S. Jin, J. Ye, M. Holland, and A. M. Rey, Suppressing the Loss of Ultracold Molecules via the Continuous Quantum Zeno Effect, Phys. Rev. Lett. 112, 070404 (2014).

[24] H. Fröml, A. Chiocchetta, C. Kollath, and S. Diehl, FluctuationInduced Quantum Zeno Effect, Phys. Rev. Lett. 122, 040402 (2019).

[25] L. D. Landau, Über die Bewegung der Elektronen in Kristallgitter, Phys. Z. Sowjetunion 3, 644 (1933).

[26] L. D. Landau and S. I. Pekar, Effective mass of a polaron, Zh. Eksp. Teor. Fiz. 18, 419 (1948) [Ukr. J. Phys. 53, 71 (2008)].

[27] H. Fröhlich, H. Pelzer, and S. Zienau, Properties of slow electrons in polar materials, London, Edinburgh, Dublin Philos. Mag. J. Sci. 41, 221 (1950).

[28] R. P. Feynman, Slow electrons in a polar crystal, Phys. Rev. 97, 660 (1955).

[29] A. Rosch and T. Kopp, Heavy Particle in a D-Dimensional Fermionic Bath: A Strong Coupling Approach, Phys. Rev. Lett. 75, 1988 (1995).

[30] F. Chevy, Universal phase diagram of a strongly interacting Fermi gas with unbalanced spin populations, Phys. Rev. A 74, 063628 (2006).

[31] R. Combescot and S. Giraud, Normal State of Highly Polarized Fermi Gases: Full Many-Body Treatment, Phys. Rev. Lett. 101, 050404 (2008).

[32] R. A. Duine and A. H. MacDonald, Itinerant Ferromagnetism in an Ultracold Atom Fermi Gas, Phys. Rev. Lett. 95, 230403 (2005).

[33] X. Cui and H. Zhai, Stability of a fully magnetized ferromagnetic state in repulsively interacting ultracold Fermi gases, Phys. Rev. A 81, 041602(R) (2010).

[34] V. Pietilä, D. Pekker, Y. Nishida, and E. Demler, Pairing instabilities in quasi-two-dimensional Fermi gases, Phys. Rev. A 85, 023621 (2012).

[35] P. Massignan, M. Zaccanti, and G. M. Bruun, Polarons, dressed molecules and itinerant ferromagnetism in ultracold Fermi gases, Rep. Prog. Phys. 77, 034401 (2014).

[36] A. Schirotzek, C.-H. Wu, A. Sommer, and M. W. Zwierlein, Observation of Fermi Polarons in a Tunable Fermi Liquid of Ultracold Atoms, Phys. Rev. Lett. 102, 230402 (2009).

[37] C. Kohstall, M. Zaccanti, M. Jag, A. Trenkwalder, P. Massignan, G. M. Bruun, F. Schreck, and R. Grimm, Metastability and coherence of repulsive polarons in a strongly interacting Fermi mixture, Nature (London) 485, 615 (2012). 
[38] M. Koschorreck, D. Pertot, E. Vogt, B. Fröhlich, M. Feld, and M. Köhl, Attractive and repulsive Fermi polarons in two dimensions, Nature (London) 485, 619 (2012).

[39] M. Sidler, P. Back, O. Cotlet, A. Srivastava, T. Fink, M. Kroner, E. Demler, and A. Imamoglu, Fermi polaron-polaritons in charge-tunable atomically thin semiconductors, Nat. Phys. 13, 255 (2017).

[40] L. B. Tan, O. Cotlet, A. Bergschneider, R. Schmidt, P. Back, Y. Shimazaki, M. Kroner, and A. Imamoglu, Interacting PolaronPolaritons, Phys. Rev. X 10, 021011 (2020).

[41] Y. Zhang, W. Ong, I. Arakelyan, and J. E. Thomas, Polaronto-Polaron Transitions in the Radio-Frequency Spectrum of a Quasi-Two-Dimensional Fermi Gas, Phys. Rev. Lett. 108, 235302 (2012).

[42] F. Tonielli, R. Fazio, S. Diehl, and J. Marino, Orthogonality Catastrophe in Dissipative Quantum Many-Body Systems, Phys. Rev. Lett. 122, 040604 (2019).

[43] W. Berdanier, J. Marino, and E. Altman, Universal Dynamics of Stochastically Driven Quantum Impurities, Phys. Rev. Lett. 123, 230604 (2019).

[44] R. Combescot, A. Recati, C. Lobo, and F. Chevy, Normal State of Highly Polarized Fermi Gases: Simple Many-Body Approaches, Phys. Rev. Lett. 98, 180402 (2007).

[45] S. Zöllner, G. M. Bruun, and C. J. Pethick, Polarons and molecules in a two-dimensional Fermi gas, Phys. Rev. A 83, 021603(R) (2011).

[46] M. M. Parish, Polaron-molecule transitions in a twodimensional Fermi gas, Phys. Rev. A 83, 051603(R) (2011).

[47] P. Kroiss and L. Pollet, Diagrammatic Monte Carlo study of quasi-two-dimensional Fermi polarons, Phys. Rev. B 90, 104510 (2014).

[48] V. Ngampruetikorn, J. Levinsen, and M. M. Parish, Repulsive polarons in two-dimensional Fermi gases, Europhys. Lett. 98, 30005 (2012).
[49] R. Schmidt, T. Enss, V. Pietilä, and E. Demler, Fermi polarons in two dimensions, Phys. Rev. A 85, 021602(R) (2012).

[50] A. Kamenev, Field Theory of Non-Equilibrium Systems (Cambridge University Press, Cambridge, 2011).

[51] L. M. Sieberer, M. Buchhold, and S. Diehl, Keldysh field theory for driven open quantum systems, Rep. Prog. Phys. 79, 096001 (2016).

[52] M. Randeria, J.-M. Duan, and L.-Y. Shieh, Bound States, Cooper Pairing, and Bose Condensation in Two Dimensions, Phys. Rev. Lett. 62, 981 (1989).

[53] J. R. Engelbrecht and M. Randeria, New Collective Mode and Corrections to Fermi-Liquid Theory in Two Dimensions, Phys. Rev. Lett. 65, 1032 (1990).

[54] J. R. Engelbrecht and M. Randeria, Low-density repulsive Fermi gas in two dimensions: Bound-pair excitations and Fermi-liquid behavior, Phys. Rev. B 45, 12419 (1992).

[55] D. K. Efimkin and A. H. MacDonald, Many-body theory of trion absorption features in two-dimensional semiconductors, Phys. Rev. B 95, 035417 (2017).

[56] M. Randeria, J.-M. Duan, and L.-Y. Shieh, Superconductivity in a two-dimensional Fermi gas: Evolution from Cooper pairing to Bose condensation, Phys. Rev. B 41, 327 (1990).

[57] E. Braaten, H.-W. Hammer, and G. P. Lepage, Open effective field theories from highly inelastic reactions, PoS (ICHEP2016) 350 (2017).

[58] P. Nozieres and S. Schmitt-Rink, Bose condensation in an attractive fermion gas: From weak to strong coupling superconductivity, J. Low Temp. Phys. 59, 195 (1985).

[59] C. Shkedrov, G. Ness, Y. Florshaim, and Y. Sagi, In situ momentum-distribution measurement of a quantum degenerate Fermi gas using Raman spectroscopy, Phys. Rev. A 101, 013609 (2020).

[60] N. Prokof'ev and B. Svistunov, Fermi-polaron problem: Diagrammatic Monte Carlo method for divergent sign-alternating series, Phys. Rev. B 77, 020408(R) (2008). 